\title{
Digital Circuit Design Utilizing Equation Solving over 'Big' Boolean Algebras
}

\author{
Ali Muhammad Ali Rushdi*, Waleed Ahmad \\ Department of Electrical and Computer Engineering \\ King Abdulaziz University \\ P. O. Box 80204, Jeddah 21589, Saudi Arabia \\ *Corresponding author: arushdi@kau.edu.sa
}

(Received June 29, 2017; Accepted September 11, 2017)

\begin{abstract}
A task frequently encountered in digital circuit design is the solution of a two-valued Boolean equation of the form $h(\boldsymbol{X}, \boldsymbol{Y}, \boldsymbol{Z})=1$, where $h: B_{2}^{k+m+n} \rightarrow B_{2}$ and $\boldsymbol{X}, \boldsymbol{Y}$, and $\boldsymbol{Z}$ are binary vectors of lengths $k, m$, and $n$, representing inputs, intermediary values, and outputs, respectively. The resultant of the suppression of the variables $\boldsymbol{Y}$ from this equation could be written in the form $g(\boldsymbol{X}, \boldsymbol{Z})=1$ where $g: B_{2}^{k+n} \rightarrow B_{2}$. Typically, one needs to solve for $\boldsymbol{Z}$ in terms of $\boldsymbol{X}$, and hence it is unavoidable to resort to 'big' Boolean algebras which are finite (atomic) Boolean algebras larger than the two-valued Boolean algebra. This is done by reinterpreting the aforementioned $g(\boldsymbol{X}, \boldsymbol{Z})$ as $g(\boldsymbol{Z}): B_{2^{K}}^{n} \rightarrow B_{2^{K}}$, where $B_{2^{K}}$ is the free Boolean algebra $F B\left(X_{1}, X_{2} \ldots \ldots . X_{k}\right)$, which has $K=2^{k}$ atoms, and $2^{K}$ elemnets. This paper describes how to unify many digital specifications into a single Boolean equation, suppress unwanted intermediary variables $\boldsymbol{Y}$, and solve the equation $g(\boldsymbol{Z})=1$ for outputs $\boldsymbol{Z}$ (in terms of inputs $\boldsymbol{X}$ ) in the absence of any information about $\boldsymbol{Y}$. The paper uses a novel method for obtaining the parametric general solutions of the 'big' Boolean equation $g(\boldsymbol{Z})=1$. The parameters used do not belong to $B_{2^{K}}$ but they belong to the two-valued Boolean algebra $B_{2}$, also known as the switching algebra or propositional algebra. To achieve this, we have to use distinct independent parameters for each asserted atom in the Boole-Shannon expansion of $g(\boldsymbol{Z})$. The concepts and methods introduced herein are demonsrated via several detailed examples, which cover the most prominent type among basic problems of digital circuit design.
\end{abstract}

Keywords- Digital design, Suppression of variables, 'Big' Boolean algebras, Boolean-equation solving, Parametric solutions.

\section{Introduction}

In a seminal work, done more than half a century ago, Ledley $(1959,1960)$ posed three 'elementary problems of digital circuit design' inspired by the arrangement in Fig. 1, which entails five quantities $\boldsymbol{X}, \boldsymbol{Y}, \boldsymbol{Z}, \boldsymbol{s}$ and $\boldsymbol{t}$ that belong to $B_{2}^{k}, B_{2}^{m}, B_{2}^{n}, B_{2}^{l}$, and $B_{2}^{l}$, respectively. This arrangement includes a 'parent' combinational network $C$ of two (vectorial) inputs $\boldsymbol{X}$ and $\boldsymbol{Y}$ and a vectorial output $\boldsymbol{t}(\boldsymbol{X}, \boldsymbol{Y})$. Network $C$ consists of two subnetworks $A$ and $B$, where subnetwork $A$ has the single (vectorial) input $\boldsymbol{X}$ and the (vectorial) output $\boldsymbol{Z}(\boldsymbol{X})$, while network $B$ has the two vectorial inputs $\boldsymbol{Z}(\boldsymbol{X})$ and $\boldsymbol{Y}$ and the (vectorial) output $\boldsymbol{s}(\boldsymbol{Z}, \boldsymbol{Y})$, which is exactly the same as the (vectorial) output $\boldsymbol{t}(\boldsymbol{X}, \boldsymbol{Y})$ of network $C$. The above arrangement involves three vectorial Boolean functions, namely $\boldsymbol{Z}(\boldsymbol{X}), \boldsymbol{s}(\boldsymbol{Z}, \boldsymbol{Y})$ and $\boldsymbol{t}(\boldsymbol{X}, \boldsymbol{Y})$. Three problems arise when one utilizes the information that $\boldsymbol{s}(\boldsymbol{Z}(\boldsymbol{X}), \boldsymbol{Y})$ and $\boldsymbol{t}(\boldsymbol{X}, \boldsymbol{Y})$ are equal, together with knowledge of two of the three functions $\boldsymbol{Z}(\boldsymbol{X}), \boldsymbol{s}(\boldsymbol{Z}, \boldsymbol{Y})$ and $\boldsymbol{t}(\boldsymbol{X}, \boldsymbol{Y})$ in order to deduce the third function. Therefore, one obtains three distinct problems, viz.

1. Type-1 problem: Given $\boldsymbol{Z}(\boldsymbol{X})$ and $\boldsymbol{s}(\boldsymbol{Z}, \boldsymbol{Y})$, find $\boldsymbol{t}(\boldsymbol{X}, \boldsymbol{Y})$.

2. Type-2 problem: Given $\boldsymbol{Z}(\boldsymbol{X})$ and $\boldsymbol{t}(\boldsymbol{X}, \boldsymbol{Y})$, find $\boldsymbol{s}(\boldsymbol{Z}, \boldsymbol{Y})$.

3. Type-3 problem: Given $\boldsymbol{s}(\boldsymbol{Z}, \boldsymbol{Y})$ and $\boldsymbol{t}(\boldsymbol{X}, \boldsymbol{Y})$, find $\boldsymbol{Z}(\boldsymbol{X})$. 
International Journal of Mathematical, Engineering and Management Sciences

Vol. 3, No. 4, 404-428, 2018

https://dx.doi.org/10.33889/IJMEMS.2018.3.4-029

Table 1 shows taxonomy of these three problems, together with an outline on how to solve them according to the scheme set by Ledley $(1959,1960)$ in his seminal work. It is obvious that the Type-1 Problem necessitates only direct substitution and hence does not warrant any further consideration. The Type-2 Problem is, in fact, the inverse problem of logic. This problem was treated extensively by nineteen-century logicians such as Jevons $(1872,1874)$, Venn (1894) and Poretsky (1898). Though interest in this problem faded away for more than half a century, it witnessed a revival at the hands of pioneers of modern digital design including Ledley (1960), Bell (1968), Cerny and Marin (1974, 1977), Cerny (1976) and Brown (1974, 1975a, 1975b), and it remains an essential element in contemporary digital design practice (Brown, 1990, 2003; Rushdi and Ba-Rukab, 2003; Steinbach and Posthoff, 2003; Baneres et al., 2009; Brown and Vranesic, 2014; Knodel et al., 2014; Rushdi, 2018b; Rushdi and Ahmad, 2018). Ledley devoted his 1959 paper entirely to the Type-3 Problem, and further illustrated it by several examples in his 1960 book, and (admirably) managed to handle it via very tedious and definitely outdated techniques. In this paper, we revisit the Type-3 Problem using four major enhancements inspired by certain new techniques, which became available only very recently, namely:

1. Instead of Ledley's representation of Boolean variables via (the now outdated) 'black-box' designation numbers and numerical Boolean matrices, we use a transparent algebraic representation all throughout, possibly aided by a pictorial interpretation via natural maps (variable-entered Karnaugh maps) (Rushdi, 1987, 2001, 2004, 2012, 2017; Rushdi and Ahmad, 2016; Rushdi and Al-Yahya, 2000, 2001; Rushdi and Amashah, 2010, 2011, 2012; Rushdi and Albarakati, 2014; Rushdi and Ba-Rukab, 2017; Rushdi, 2018a; Ahmad and Rushdi, 2018).

2. We do not follow exactly the scheme outlined in Table 1 . We do not separate our requirements into an antecedence requirement $(\boldsymbol{s} \rightarrow \boldsymbol{t})$ and a consequence requirement $(\boldsymbol{t} \rightarrow \boldsymbol{s})$, but instead combine these two requirements into a single requirement $(\boldsymbol{s} \leftrightarrow \boldsymbol{t})=(\boldsymbol{s} \odot \boldsymbol{t})$. Therefore, we end up with a single equation to solve

$$
h(\boldsymbol{X}, \boldsymbol{Y}, \boldsymbol{Z})=\bigwedge_{i=1}\left(s_{i}(\boldsymbol{Z}, \boldsymbol{Y}) \odot t_{i}(\boldsymbol{X}, \boldsymbol{Y})\right)=1 .
$$

3. We do not solve the aforementioned equation to obtain values of independent variables $\boldsymbol{X}, \boldsymbol{Y}$ and $\boldsymbol{Z}$ in $B_{2}^{k}, B_{2}^{m}, B_{2}^{n}$, respectively, but instead we solve for the dependent variables $\boldsymbol{Z}$ in terms of the independent variables $\boldsymbol{X}$, and $\boldsymbol{Y}$. This means that instead of viewing the function $h$ above as $h: B_{2}^{k+m+n} \rightarrow B_{2}$, we treat it as $h: B^{n} \rightarrow B$, where $B$ is a 'big' Boolean algebra equivalent to the free Boolean algebra $F B(\boldsymbol{X}, \boldsymbol{Y})$. As such, the roles of $\boldsymbol{X}$ and $\boldsymbol{Y}$ as variables is relegated to those of generators. Since $B$ has $(k+m)$ scalar generators, it has $2^{(k+m)}$ atoms and hence $2^{2^{(k+m)}}$ elements. The task of solving $h(\boldsymbol{X}, \boldsymbol{Y}, \boldsymbol{Z})=1$ over such an algebra is facilitated by a two-century effort that culminated recently in a technique for a compact listing of all particular solutions of any 'big' Boolean equation (Rushdi and Ahmad, 2017a, 2017b).

4. We avoid the task of extensive search among the solutions of $h(\boldsymbol{X}, \boldsymbol{Y}, \boldsymbol{Z})=1$ for those solutions that interrelate $\boldsymbol{Z}$ and $\boldsymbol{X}$ independently of $\boldsymbol{Y}$. Instead, we employ a technique, recently developed by Brown (2011), that suppresses the undesired variables $\boldsymbol{Y}$ in the equation $h(\boldsymbol{X}, \boldsymbol{Y}, \boldsymbol{Z})=1$, replacing that equation by another $g(\boldsymbol{X}, \boldsymbol{Z})=1$, where $g$ is not directly interpreted as $g: B_{2}^{k+n} \rightarrow B_{2}$ but is interpreted as $g: B_{2^{K}}^{n} \rightarrow B_{2^{K}}$, where $B_{2^{K}}$ is a 'big' Boolean algebra of $K=2^{k}$ atoms and $2^{K}$ elements constructed as $F B(\boldsymbol{X})=F B\left(X_{1}, X_{2} \ldots \ldots X_{k}\right)$. 
International Journal of Mathematical, Engineering and Management Sciences

Vol. 3, No. 4, 404-428, 2018

https://dx.doi.org/10.33889/IJMEMS.2018.3.4-029

According to Brown's technique the equation $g(\boldsymbol{X}, \boldsymbol{Z})=1$ is constructed such as to have exactly all the solutions for $\boldsymbol{Z}$ in terms of $\boldsymbol{X}$ that are independent of $\boldsymbol{Y}$.

The organization of the remainder of this paper is as follows. Section 2 reviews our method for handling Ledley's Type-3 Problem of digital design. The section explains how to unify given digital specifications (such as the $\boldsymbol{s}=\boldsymbol{t}$ one) into a single Boolean equation. It also summarizes the method of suppression of variables, derives parametric solutions of any Boolean equation such that they can be recast into a very compact listing of all particular solutions, which enables one to easily locate particular solutions of certain desirable features. Section 3 illustrates the mathematical details of our proposed method by applying it to five examples of Ledley (1959, 1960). This section demonstrates clearly the dramatic advantages of the method including its conceptual clarity, high speed, avoidance of unwarranted cumbersome tasks, and better control on outcomes. These advantages do not pertain only to pedagogical issues, but also suggest some enhancements of contemporary practice of digital design. The paper is concluded in Section 4.

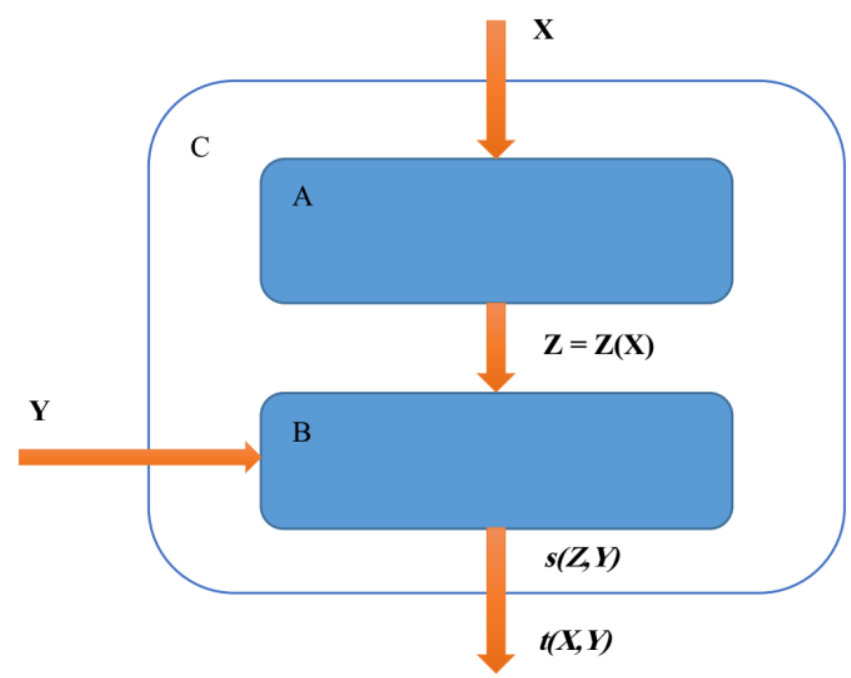

Fig. 1. An outline of digital networks pertaining to the three elementary problems of digital design posed by Ledley $(1959,1960)$

Table 1. Taxonomy of Ledley's elementary problems of digital design

\begin{tabular}{|c|c|c|c|c|}
\hline Problem & Given & & Find & How \\
\hline Type 1 & $Z(X)$ & $s(Z, Y)$ & $t(X, Y)$ & $\boldsymbol{t}(\boldsymbol{X}, \boldsymbol{Y})=\boldsymbol{s}(\boldsymbol{Z}(\boldsymbol{X}), \boldsymbol{Y})$, i.e., just direct and straightforward substitution. \\
\hline Type 2 & $Z(X)$ & $t(X, Y)$ & $s(Z, Y)$ & $\begin{array}{l}\text { *Solve for } \boldsymbol{X}(\boldsymbol{Z}), \text { i.e., perform logical inversion (solve an inverse problem of logic). } \\
* \boldsymbol{s}(\boldsymbol{Z}, \boldsymbol{Y})=\boldsymbol{t}(\boldsymbol{X}(\boldsymbol{Z}), \boldsymbol{Y})\end{array}$ \\
\hline Type 3 & $s(Z, Y)$ & $t(X, Y)$ & $Z(X)$ & $\begin{array}{l}\text { *Find antecedence solutions } \boldsymbol{Z}_{\boldsymbol{a}}(\boldsymbol{X}) \text { such that } \boldsymbol{s}_{\boldsymbol{a}}\left(\boldsymbol{Z}_{\boldsymbol{a}}(\boldsymbol{X}), \boldsymbol{Y}\right) \rightarrow \boldsymbol{t}(\boldsymbol{X}, \boldsymbol{Y}) \text {. } \\
\text { *Find consequence solutions } \boldsymbol{Z}_{\boldsymbol{c}}(\boldsymbol{X}) \text { such that } \boldsymbol{t}(\boldsymbol{X}, \boldsymbol{Y}) \rightarrow \boldsymbol{s}_{\boldsymbol{c}}=\boldsymbol{s}\left(\boldsymbol{Z}_{\boldsymbol{c}}(\boldsymbol{X}), \boldsymbol{Y}\right) \text {. } \\
\text { *Find the intersection of the sets of antecedence solutions and consequence solutions, so } \\
\text { as to obtain solutions satisfying }((\boldsymbol{s} \rightarrow \boldsymbol{t}) \cap(\boldsymbol{t} \rightarrow \boldsymbol{s})) \leftrightarrow(\boldsymbol{s} \odot \boldsymbol{t}) \leftrightarrow \bigwedge_{i=1}^{n}\left(s_{i} \odot t_{i}\right)=1 \text {. } \\
\text { *Obtain solutions of this equation that specify values of } \boldsymbol{Z}, \boldsymbol{X}, \boldsymbol{Y} \text { in } B_{2} \text {. } \\
\text { *Select solutions that relate } \boldsymbol{Z} \text { to } \boldsymbol{X} \text { independently of } \boldsymbol{Y} \text {. }\end{array}$ \\
\hline
\end{tabular}


International Journal of Mathematical, Engineering and Management Sciences

Vol. 3, No. 4, 404-428, 2018

https://dx.doi.org/10.33889/IJMEMS.2018.3.4-029

\section{Steps of the Method}

\subsection{Unifying Specifications into a Single Boolean Equation}

Without loss of generality, let us assume that the digital system at hand is specified by the system of $l$ Boolean equations (suggested by Fig. 1) of the form

$s=t$

or equivalently

$s_{i}=t_{i}, \quad 1 \leq i \leq l$

where $s_{i}=s_{i}(\mathbf{X}, \mathbf{Y}, \mathbf{Z})$ and $t_{i}=t_{i}(\mathbf{X}, \mathbf{Y}, \mathbf{Z})$, and occasionally we might have $t_{i}=0$ or $t_{i}=1$. The system (1) of $l$ scalar equations reduces to a single Boolean equation of the form

$h(\boldsymbol{X}, \boldsymbol{Y}, \boldsymbol{Z})=1$

where

$h(\boldsymbol{X}, \boldsymbol{Y}, \boldsymbol{Z}) \equiv \wedge_{i=1}^{l}\left(s_{i} \odot t_{i}\right)$

or of the form

$r(\boldsymbol{X}, \boldsymbol{Y}, \boldsymbol{Z})=0$

where

$r(\boldsymbol{X}, \boldsymbol{Y}, \boldsymbol{Z})=\bar{h}(\boldsymbol{X}, \boldsymbol{Y}, \boldsymbol{Z}) \equiv \mathrm{\vee}_{i=1}^{l}\left(s_{i} \oplus t_{i}\right)$

The symbols $\Lambda, V, \oplus$, and $\odot$ in Equations (2b) and (3b) depict the AND operator, the OR operator, the XOR (Exclusive-OR) operator and the XNOR (coincidence or equivalence) operator, respectively, defined as shown in Table 2. The relation between (2b) and 3(b) is just an expression of the two De' Morgan's laws. Note that the AND and OR operators are dual ones, while the XNOR and XOR operators are both complementary and dual ones.

Table 2. Definition of binary Boolean operators in equations (2b) and (3b)

\begin{tabular}{|c|c|c|c|c|c|}
\hline$s_{i}$ & $t_{i}$ & $s_{i} \wedge t_{i}$ & $s_{i} \vee t_{i}$ & $s_{i} \odot t_{i}$ & $s_{i} \oplus t_{i}$ \\
\hline 0 & 0 & 0 & 0 & 1 & 0 \\
\hline 0 & 1 & 0 & 1 & 0 & 1 \\
\hline 1 & 0 & 0 & 1 & 0 & 1 \\
\hline 1 & 1 & 1 & 1 & 1 & 0 \\
\hline
\end{tabular}

\subsection{Suppression of Variables}

Brown (2011) proved that the resultant of suppression of the variables $\boldsymbol{Y}$ from the Boolean equation (3a) (called the parent equation) is the derived Boolean equation

$f(\boldsymbol{X}, \boldsymbol{Z})=0$

where

$f(\boldsymbol{X}, \boldsymbol{Z}) \equiv \mathrm{\vee}_{\mathbf{A} \in\{0,1\}^{m}} \quad r(\boldsymbol{X}, \boldsymbol{A}, \boldsymbol{Z})$ 
International Journal of Mathematical, Engineering and Management Sciences

Vol. 3, No. 4, 404-428, 2018

https://dx.doi.org/10.33889/IJMEMS.2018.3.4-029

and that the solutions of the derived equation (4a) are exactly those of the parent equation (3a) that do not involve the suppressed variables $\boldsymbol{Y}$.

We will herein utilize the dual of the above result, namely that if we use (2a) instead of (3a) as a parent equation, then the resultant of suppression of the variables $\boldsymbol{Y}$ is now the complementary derived Boolean equation

$g(\boldsymbol{X}, \boldsymbol{Z})=1$

where

$g(\boldsymbol{X}, \boldsymbol{Z}) \equiv \wedge_{\mathbf{A} \in\{0,1\}^{m}} h(\boldsymbol{X}, \boldsymbol{A}, \boldsymbol{Z})$

and the solutions of the derived equation (5a) are exactly those of the parent equation (2a) that do not involve the suppressed variables $\boldsymbol{Y}$.

\subsection{Derivation of Parametric Solutions}

We seek solutions of the Boolean equation

$g(\boldsymbol{X}, \boldsymbol{Z})=1$

where $g(\boldsymbol{X}, \boldsymbol{Z}): B_{2}^{k+n} \rightarrow B_{2}$, is a two-valued Boolean function of $k$ two-valued variables $\boldsymbol{X}=$ $\left[\begin{array}{llll}X_{1} & X_{2} & \ldots & X_{k}\end{array}\right]^{T}$ and $n$ two-valued variables $\boldsymbol{Z}=\left[\begin{array}{llll}Z_{1} & Z_{2} & \ldots & Z_{n}\end{array}\right]^{T}$. However, we do not need a listing of binary solutions for $\boldsymbol{X}$ and $\boldsymbol{Z}$, but instead we want to express $\boldsymbol{Z}$ in terms of $\boldsymbol{X}$. This is a prominent case when the use of 'big' Boolean algebras (ones other than the two-valued algebras) is unavoidable. We view $g(\boldsymbol{X}, \boldsymbol{Z})$ as $g(\boldsymbol{X} ; \boldsymbol{Z})$ or simply $g(\boldsymbol{Z})$ and rewrite (1) as

$g(Z)=1$

where $g(\boldsymbol{Z}): B_{2^{K}}^{n} \rightarrow B_{2^{K}}$, and $B_{2^{K}}$ is the free Boolean algebra $F B\left(X_{1}, X_{2} \ldots \ldots X_{k}\right)$ with $K=2^{k}$ atoms and $2^{K}$ elements. Now we express $g(\boldsymbol{Z})$ by its Minterm Canonical Form (MCF) (Brown, 1990)

$g(\boldsymbol{Z}) \equiv \mathbf{V}_{\mathbf{A} \in\{0,1\}^{n}} g(\boldsymbol{A}) \boldsymbol{Z}^{\boldsymbol{A}}$

For $\boldsymbol{Z}=\left[\begin{array}{llll}Z_{1} Z_{2} & \ldots & Z_{n}\end{array}\right]^{T} \in B_{2^{K}}^{n}, \boldsymbol{A}=\left[\begin{array}{llll}a_{1} & a_{2} \ldots & a_{n}\end{array}\right]^{T} \in\{0,1\}^{n}$, the symbol $\boldsymbol{Z}^{\boldsymbol{A}}$ is defined as

$Z^{A}=Z_{1}^{a_{1}} Z_{2}^{a_{2}} \ldots Z_{n}^{a_{n}}$

where $Z_{i}{ }^{a_{i}}$ takes the value $\bar{Z}_{i}$ (complemented literal) if $a_{i}=0$, and takes the value $Z_{i}$ (uncomplemented literal) if $a_{i}=1$. For $\boldsymbol{A} \in\{0,1\}^{n}$, the symbol $\boldsymbol{Z}^{\boldsymbol{A}}$ spans the minterms of $\boldsymbol{Z}$, which are the $2^{n}$ elementary or primitive products

$\overline{Z_{1}} \overline{Z_{2}} \ldots \overline{Z_{n-1}} \overline{Z_{n}}, \quad \overline{Z_{1}} \overline{Z_{2}} \ldots \overline{Z_{n-1}} Z_{n}, \ldots, Z_{1} Z_{2} \ldots Z_{n-1} Z_{n}$

The constant values $g(\boldsymbol{A})$ in equation (7) are elements of $B_{2^{K}}$ called the discriminants of $g(\boldsymbol{Z})$. These discriminants are the entries of the natural map of $g(\boldsymbol{Z})$ which has an input domain $\{0,1\}^{n} \subseteq$ $B_{2^{K}}^{n}$. The Boolean algebra $B_{2^{K}}=F B\left(X_{1}, X_{2} \ldots \ldots . X_{k}\right)$, has generators $X_{i}(1 \leq i \leq k)$ which 
International Journal of Mathematical, Engineering and Management Sciences

Vol. 3, No. 4, 404-428, 2018

https://dx.doi.org/10.33889/IJMEMS.2018.3.4-029

look like variables (In fact, they were originally our input variables before we changed their roles to generators). Therefore, we can accept the name assigned (for historical reasons) to the natural map of $g(\boldsymbol{Z})$, namely the name of the Variable-Entered Karnaugh Map (VEKM). We now observe that the minterms of $\boldsymbol{X}$, which are the $2^{k}=K$ elementary or primitive products

$\overline{X_{1}} \overline{X_{2}} \ldots \overline{X_{k-1}} \overline{X_{k}}, \quad \overline{X_{1}} \overline{X_{2}} \ldots \overline{X_{k-1}} X_{k}, \ldots, X_{1} X_{2} \ldots X_{k-1} X_{k}$

are exactly the atoms of the underlying Boolean algebra. For convenience, we call these atoms $T_{i}$ $(0 \leq i \leq(K-1))$, and hence $g(\boldsymbol{A})$ can be written as

$g(\boldsymbol{A})=\mathrm{\vee}_{i=0}^{K-1}\left(e_{i}(\boldsymbol{A}) \wedge T_{i}\right)$

where we use the symbol $e_{i}(\boldsymbol{A})$ to denote an indicator of the event that atom $T_{i}$ appears in the expression of $g(\boldsymbol{A})$, i.e.,

$$
e_{i}(\boldsymbol{A})=\left\{\begin{array}{l}
1, \text { if } T_{i} \rightarrow g(\boldsymbol{A}) \\
0, \text { otherwise }
\end{array}\right\}=g(\boldsymbol{A}) / T_{i}
$$

where the symbol $(\boldsymbol{r} / \boldsymbol{s})=(\boldsymbol{r})_{s=1}$ denotes the Boolean quotient of $\boldsymbol{r}$ by $\boldsymbol{s}$ (Brown, 1990). Equation (12) means that $e_{i}(\boldsymbol{A})$ indicates whether atom $T_{i}$ appears in the cell $\boldsymbol{A}$ of the natural map for $g(\boldsymbol{Z})$. Now, we define $n_{i}\left(0 \leq n_{i} \leq 2^{n}\right)$ as the total number of actual appearances of $T_{i}$ in the expression (11) for $g(\boldsymbol{A})$, i.e.,

$n_{i}=\sum_{\mathrm{A} \in\{0,1\}^{n}} e_{i}(\boldsymbol{A})$

The total number $N_{\text {unconditional }}$ of unconditional particular solutions of (1a) over $B_{2^{K}}$ (as it is) is given by

$N_{\text {unconditional }}=\prod_{i=0}^{K-1} n_{i}$

This number is zero if some $n_{i}=0$, i.e., if an atom $T_{i}$ never makes its way to any expression $g(\boldsymbol{A})$ where $\boldsymbol{A} \in\{0,1\}^{n}$ (i.e., if $T_{i}$ does not appear in any cell of the map for $g(\boldsymbol{Z})$ ). To avoid such a situation, one must insist on the consistency condition that any atom $T_{i}$ such that $n_{i}=0$ must be forbidden or nullified. This means that the underlying Boolean algebra loses these atoms and hence collapses to a smaller algebra, i.e., to one of its strict sub algebras. The number of solutions over this new Boolean algebra is

$N_{\text {conditional }}=\prod_{\substack{i=0 \\ n_{i} \neq 0}}^{K-1} n_{i}$

Now we introduce a set of parameters $\boldsymbol{p}_{\boldsymbol{i}}\left(0 \leq i \leq(K-1), n_{i} \neq 0\right)$ to construct an orthonormal set of tags to attach to instances of appearances of the asserted atom $T_{i}$ in the discriminants $g(\boldsymbol{A})$ (i.e., in the cells $\boldsymbol{A} \in\{0,1\}^{n}$ of the natural map of $g(\boldsymbol{Z})$ ). The number of parameters for atom $T_{i}$ (the length of vector $\boldsymbol{p}_{\boldsymbol{i}}$ ) is given by

$l\left(\boldsymbol{p}_{i}\right)=\left[\log _{2} n_{i}\right], \quad 0 \leq i \leq(K-1), n_{i} \neq 0$ 
International Journal of Mathematical, Engineering and Management Sciences

Vol. 3, No. 4, 404-428, 2018

https://dx.doi.org/10.33889/IJMEMS.2018.3.4-029

Here, $\lceil x\rceil$ denotes the ceiling of the real number $x$, i.e., the smallest integer greater than or equal to $x$. The parameters $\boldsymbol{p}_{\boldsymbol{i}}$ can be used to generate a set of $n_{i} \leq 2^{l\left(\boldsymbol{p}_{\boldsymbol{i}}\right)}$ orthonormal tags $\left\{t_{1}, t_{2} \ldots t_{n_{i}}\right\}$, such that

$t_{1} \vee t_{2} \vee \ldots \vee t_{n_{i}}=1$

$t_{j_{1}} \wedge t_{j_{2}}=0 \quad \forall j_{1}, j_{2} \in\left\{1,2, \ldots, n_{i}\right\}$

When $n_{i}=2^{l\left(\boldsymbol{p}_{\boldsymbol{i}}\right)}$ the set of orthonormal tags can be visualized as the products of cells in a Karnaugh map whose map variables are the underlying parameters. If $2^{l\left(\boldsymbol{p}_{\boldsymbol{i}}\right)-1}<n_{i}<2^{l\left(\boldsymbol{p}_{\boldsymbol{i}}\right)}$, some cells of such a map are merged, and the map reduces to a map-like structure.

When each appearance of an atom $T_{i}$ is tagged by a particular member of its orthonormal set of tags, an auxiliary function $G\left(\boldsymbol{Z}, \boldsymbol{p}_{\boldsymbol{i}}\right)\left(0 \leq i \leq(K-1), n_{i} \neq 0\right)$ results. The parametric solution is now given by (Brown, 1970, 2003; Rushdi and Amashah, 2011).

$Z_{u}=\mathrm{v}_{\left\{\mathbf{A} \in\{0,1\}^{n} \mid A_{u}=1\right\}} G\left(\boldsymbol{A}, \boldsymbol{p}_{i}\right) .1 \leq u \leq n,\left(0 \leq i \leq(K-1), n_{i} \neq 0\right)$

The total number of parameters used in (18) to construct the tags for all atoms is given by

$E=\sum_{i=1}^{k} l\left(\boldsymbol{p}_{\boldsymbol{i}}\right)=\sum_{i=1}^{k}\left\lceil\log _{2}\left(n_{i}\right)\right\rceil$

The conventional method is to select the parameter vectors from a shared pool of parameters so as to minimize the number of parameters used, which then becomes

$E^{\prime}=\max _{i} l\left(\boldsymbol{p}_{i}\right)=\max _{i}\left\lceil\log _{2} n_{i}\right\rceil=\left\lceil\log _{2}\left(\max _{i} n_{i}\right)\right\rceil$

However, parameters used must then belong to the underlying Boolean algebra (possibly collapsed due to the consistency condition). We now propose to use independent parameters $\boldsymbol{p}_{\boldsymbol{i}}$ for each atom $T_{i}\left(0 \leq i \leq K-1, n_{i} \neq 0\right)$. The expressions (18) will not be as compact as they are in the conventional case, but the independent parameters $\boldsymbol{p}_{\boldsymbol{i}}$ now belong to the two-valued Boolean algebra $B_{2}$ (Brown, 2003; Rushdi and Amashah, 2011), a fact that facilitates the generation of all particular solutions as will be seen shortly in the next subsection.

\subsection{Listing of All Particular Solutions}

The parametric solutions (18) can be used to generate all particular solutions through the use of an expansion tree. Generally, in the conventional method, this expansion tree is a complete tree that entails the assignment of $2^{K^{\prime}}$ values to each of $E^{\prime}$ parameters where $K^{\prime} \leq K$ is the final number of atoms of the underlying Boolean algebra (possibly after some collapse due to the consistency condition). Each parent node has $2^{K^{\prime}}$ children nodes and the tree has $E^{\prime}$ levels beyond its root. Therefore, the tree has $\left(2^{K^{\prime}}\right)^{E^{\prime}}=2^{K^{\prime} E^{\prime}}$ leaves. These leaves constitute the whole set of particular solutions, possibly with repetitions. However, to avoid repetitions, we make sure, right from the first expansion level, to combine any sibling nodes that share the same solution value. With this kind of combining, the tree ceases to be a complete one, and its leaves become exactly the particular solutions, i.e., without repetitions. If we further allow combining cousin 
International Journal of Mathematical, Engineering and Management Sciences

Vol. 3, No. 4, 404-428, 2018

https://dx.doi.org/10.33889/IJMEMS.2018.3.4-029

(same-level) nodes, the tree is replaced by an acyclic graph that lists particular solution compactly (Rushdi, 2012).

In the method proposed herein, a complete version of the expansion tree requires the assignment of binary values $\{0,1\}$ to each of $E$ independent parameters. Since the complete binary tree has $E$ levels beyond its root, it has $2^{E}$ leaves. With merging of sibling nodes of equal solution values, the tree is no longer complete, and its leaves are just the particular solutions without repetitions. The size of the expansion tree in the proposed method is typically less than that in the conventional method since typically $E<K^{\prime} E^{\prime}$ (though $E>E^{\prime}$ ). However, the true advantage of the proposed method is that it allows us to avoid the use of an expansion tree (or an expansion acyclic graph) altogether. The key to this is the observation that the parametric solution (18) can be rewritten as the weighted sum of the atoms $T_{i}$ that appear in the discriminants $g(\boldsymbol{A})$ (as expressed in (11)) of the function $g(\boldsymbol{Z})$, viz.

$\boldsymbol{Z}=\underset{\substack{i=0 \\ n_{i} \neq 0}}{K-1}\left(\boldsymbol{C o}\left(T_{i}\right) \wedge T_{i}\right)$

where we call the vector $\boldsymbol{C o}\left(T_{i}\right)$ the 'contribution' of the asserted atom $T_{i}$ and call the conjunction $\left(\boldsymbol{C o}\left(T_{i}\right) \wedge T_{i}\right)$ the 'total contribution' of that atom. We now note that $\boldsymbol{C o}\left(T_{i}\right)\left\{\right.$ or $\left.\boldsymbol{C o}\left(T_{i}\right) \wedge T_{i}\right\}$ has exactly $n_{i}$ possible values, which can be conveniently listed via the same Karnaugh-map-like structure used in the representation of the associated tags. Therefore, we interpret (21) as a method of conveniently listing all particular solutions as a disjunction of total contributions of asserted atoms $T_{i}$, where the total contribution is given in all its $n_{i}$ possibilities. To obtain a specific particular solution, one has simply to pick up arbitrarily one of the possibilities of the total contribution for every atom, and then add the selected total contributions together. The total number of particular solutions obtained this way agrees with that given by (15).

\subsection{Picking up a Particular Solution of Specific Features}

Equation (21) is of a paramount importance, as it provides a listing of a (possibly huge) number of all particular solutions in a compact space. As such, it allows picking up certain solutions enjoying particular desirable features simply by a quick inspection of the aforementioned listing. This point will be clarified further by way of examples in the next section.

\section{Examples}

In the following, we illustrate the method of Section 2 by revisiting five Type-3 examples handled by Ledley $(1959,1960)$. In each of these, we recover the results of Ledley in a much faster, more systematic and transparent, and less error-prone way. In one particular case, we resolve and circumvent a certain discrepancy that Ledley's technique fell short of handling completely.

\section{Example 1}

This example was studied before by Ledley (1960) and Brown (2011). For this example, the $\boldsymbol{s}$ and $\boldsymbol{t}$ functions are scalars of the form

$$
\begin{aligned}
& s=\bar{Z}_{1} Z_{3} \vee Y_{1} Z_{1} \bar{Z}_{2} \vee Y_{2} Z_{2} \bar{Z}_{3} \\
& t=X_{1} X_{2} \vee \bar{X}_{2} X_{3} Y_{1} \vee X_{1} \bar{X}_{3} Y_{2} \vee X_{2} Y_{2}
\end{aligned}
$$


International Journal of Mathematical, Engineering and Management Sciences

Vol. 3, No. 4, 404-428, 2018

https://dx.doi.org/10.33889/IJMEMS.2018.3.4-029

where

$s=t$.

Equation (22c) can be reduced into an equation similar to (2a) of the form (2b), viz.,

$h(\boldsymbol{X}, \boldsymbol{Y}, \boldsymbol{Z})=s \odot t$

Fig. 2 is an expression of $h(\boldsymbol{X}, \boldsymbol{Y}, \boldsymbol{Z})$ in terms of a variable-entered Karnaugh map (VEKM) of map variables $Y_{1}$ and $Y_{2}$. The map has entries in terms of the $\odot$ operator in Fig. 2(a). These terms are rewritten with the $\odot$ operator replaced by its POS form in Fig. 2(b). The $Y$ variables are suppressed via (5b) by ANDing the entries in Fig. 2(b), namely

$$
\begin{aligned}
& g(\boldsymbol{X}, \boldsymbol{Z})=\left(\bar{Z}_{1} Z_{3} \vee \bar{X}_{1} \vee \bar{X}_{2}\right)\left(\bar{Z}_{1} Z_{3} \vee Z_{1} \bar{Z}_{2} \vee \bar{X}_{1} X_{2} \vee \bar{X}_{2} \bar{X}_{3}\right)\left(\bar{Z}_{1} Z_{3} \vee Z_{2} \bar{Z}_{3} \vee \bar{X}_{1} \bar{X}_{2} \vee\right. \\
& \left.\bar{X}_{2} X_{3}\right)\left(\bar{Z}_{1} Z_{3} \vee Z_{1} \bar{Z}_{2} \vee Z_{2} \bar{Z}_{3} \vee \bar{X}_{1} \bar{X}_{2} \bar{X}_{3}\right)\left(Z_{1} \vee \bar{Z}_{3} \vee X_{1} X_{2}\right)\left(\bar{Z}_{1} \bar{Z}_{3} \vee Z_{1} Z_{2} \vee X_{1} X_{2} \vee\right. \\
& \left.\bar{X}_{2} X_{3}\right)\left(Z_{1} Z_{3} \vee \bar{Z}_{2} \bar{Z}_{3} \vee X_{1} \bar{X}_{3} \vee X_{2}\right)\left(\bar{Z}_{1} \bar{Z}_{2} \bar{Z}_{3} \vee Z_{1} Z_{2} Z_{3} \vee X_{1} \vee X_{2} \vee X_{3}\right)
\end{aligned}
$$

The function $g(\boldsymbol{X} ; \boldsymbol{Z})$ is computed via its natural map of map variables $Z_{1}, Z_{2}$, and $Z_{3}$ in Fig. $3 \mathrm{a}$. The final result of the computation is shown in Fig. $3 b$ which is again redrawn as Fig. $3 \mathrm{c}$ such that every discriminant (cell entry) of $g(\boldsymbol{X} ; \boldsymbol{Z})$ is given as a minterm expansion (disjunction of atoms). Each of the eight atoms of $F B\left(X_{1}, X_{2}, X_{3}\right)$ appears twice in Fig. 3c, which means that the consistency condition is the identity

$0=0$

and the total number of particular solutions is

$N=2^{8}=256$

Each atom $T_{i}$ requires a single parameter $p_{i}$ to produce a set of orthonormal tags $\left\{p_{i}, \bar{p}_{i}\right\}$. The corresponding auxiliary function $G(\boldsymbol{X} ; \boldsymbol{Z}, \boldsymbol{p})$ is shown in Fig. 4. The parametric solution is then given by either of the following equations

$$
\begin{aligned}
& {\left[\begin{array}{l}
Z_{1} \\
Z_{2} \\
Z_{3}
\end{array}\right]=\left[\begin{array}{c}
T_{0} p_{0} \vee T_{1} \vee T_{2} p_{2} \vee T_{3} p_{3} \vee T_{4} p_{4} \vee T_{5} \\
T_{0} p_{0} \vee T_{2} \vee T_{3} \vee T_{4} \vee T_{6} p_{6} \vee T_{7} p_{7} \\
T_{0} p_{0} \vee T_{1} p_{1} \vee T_{5} p_{5} \vee T_{6} \vee T_{7}
\end{array}\right]} \\
& {\left[\begin{array}{l}
Z_{1} \\
Z_{2} \\
Z_{3}
\end{array}\right]=\left[\begin{array}{c}
\bar{X}_{1} \bar{X}_{2} \bar{X}_{3} p_{0} \vee \bar{X}_{2} X_{3} \vee \bar{X}_{1} X_{2} \bar{X}_{3} p_{2} \vee \bar{X}_{1} X_{2} X_{3} p_{3} \vee X_{1} \bar{X}_{2} \bar{X}_{3} p_{4} \\
\bar{X}_{1} \bar{X}_{2} \bar{X}_{3} p_{0} \vee \bar{X}_{1} X_{2} \vee X_{1} \bar{X}_{2} \bar{X}_{3} \vee X_{1} X_{2} \bar{X}_{3} p_{6} \vee X_{1} X_{2} X_{3} p_{7} \\
\bar{X}_{1} \bar{X}_{2} \bar{X}_{3} p_{0} \vee \bar{X}_{1} \bar{X}_{2} X_{3} p_{1} \vee X_{1} \bar{X}_{2} X_{3} p_{5} \vee X_{1} X_{2}
\end{array}\right]}
\end{aligned}
$$

where the symbols $T_{i}=\boldsymbol{X}^{\boldsymbol{I}}$ denote the eight atoms of $F B\left(X_{1}, X_{2}, X_{3}\right)$ and $\boldsymbol{I}=\left(i_{1} i_{2} i_{3}\right)$ expresses the integer $i$ in binary notation $\left(i=4 i_{1}+2 i_{2}+i_{3}\right)$. Fig. 5 displays all 256 particular solutions of this example. Each particular solution is a disjunction of possible contributions of atoms $T_{i}(0 \leq$ $i \leq 7$ ). Here the contribution of each atom is represented by a single-variable (2-cell) Karnaugh map. As an example of identifying one particular solution (without any deliberate effort to achieve compactness), we select the possible contribution in the top cell for each atom. Hence, we obtain 
International Journal of Mathematical, Engineering and Management Sciences

Vol. 3, No. 4, 404-428, 2018

https://dx.doi.org/10.33889/IJMEMS.2018.3.4-029

$\left[\begin{array}{c}Z_{1} \\ Z_{2} \\ Z_{3}\end{array}\right]=\left[\begin{array}{c}\bar{X}_{2} X_{3} \\ \bar{X}_{1} X_{2} \vee X_{1} \bar{X}_{2} \bar{X}_{3} \\ X_{1} X_{2}\end{array}\right]$

An obvious slight improvement is possible if we use the lower-cell contribution of $T_{6}=$ $X_{1} X_{2} \bar{X}_{3}$, instead of the upper-cell one, to obtain

$\left[\begin{array}{c}Z_{1} \\ Z_{2} \\ Z_{3}\end{array}\right]=\left[\begin{array}{c}\bar{X}_{2} X_{3} \\ \bar{X}_{1} X_{2} \vee X_{1} \bar{X}_{3} \\ X_{1} X_{2}\end{array}\right]$

A better solution was found via informal means by Ledley (1960); and re-obtained by branchand-bound search (Brown, 2011). This solution is

$$
\begin{aligned}
& {\left[\begin{array}{c}
\bar{X}_{2} \\
X_{2} \vee \bar{X}_{3} \\
\bar{X}_{1} \bar{X}_{2} \vee X_{1} X_{2}
\end{array}\right]=\left[\begin{array}{l}
\bar{X}_{1} \bar{X}_{2} \bar{X}_{3} \\
\bar{X}_{1} \bar{X}_{2} \bar{X}_{3} \\
\bar{X}_{1} \bar{X}_{2} \bar{X}_{3}
\end{array}\right] \vee\left[\begin{array}{c}
\bar{X}_{1} \bar{X}_{2} X_{3} \\
0 \\
\bar{X}_{1} \bar{X}_{2} X_{3}
\end{array}\right] \vee\left[\begin{array}{c}
0 \\
\bar{X}_{1} X_{2} \bar{X}_{3} \\
0
\end{array}\right] \vee\left[\begin{array}{c}
0 \\
\bar{X}_{1} X_{2} X_{3} \\
0
\end{array}\right] \vee\left[\begin{array}{c}
X_{1} \bar{X}_{2} \bar{X}_{3} \\
X_{1} \bar{X}_{2} \bar{X}_{3} \\
0
\end{array}\right] \vee\left[\begin{array}{c}
X_{1} \bar{X}_{2} X_{3} \\
0 \\
0
\end{array}\right] \mathrm{v}} \\
& {\left[\begin{array}{c}
0 \\
X_{1} X_{2} \bar{X}_{3} \\
X_{1} X_{2} \bar{X}_{3}
\end{array}\right] \vee\left[\begin{array}{c}
0 \\
X_{1} X_{2} X_{3} \\
X_{1} X_{2} X_{3}
\end{array}\right]}
\end{aligned}
$$

\begin{tabular}{|c|c|}
\hline $\bar{Z}_{1} Z_{3} \odot X_{1} X_{2}$ & $\left(\bar{Z}_{1} Z_{3} \vee Z_{1} \bar{Z}_{2}\right) \odot\left(X_{1} X_{2} \vee \bar{X}_{2} X_{3}\right)$ \\
\hline$\left(\bar{Z}_{1} Z_{3} \vee Z_{2} \bar{Z}_{3}\right) \odot\left(X_{1} X_{2} \vee X_{1} \bar{X}_{3} \vee X_{2}\right)$ & $\begin{array}{l}\left(\bar{Z}_{1} Z_{3} \vee Z_{1} \bar{Z}_{2} \vee Z_{2} \bar{Z}_{3}\right) \odot\left(X_{1} X_{2} \vee \bar{X}_{2} X_{3} \vee\right. \\
\left.X_{1} \bar{X}_{3} \vee X_{2}\right)\end{array}$ \\
\hline
\end{tabular}

(a) $\quad h(\boldsymbol{X}, \boldsymbol{Y}, \boldsymbol{Z})$ in terms of the $\odot$ operator

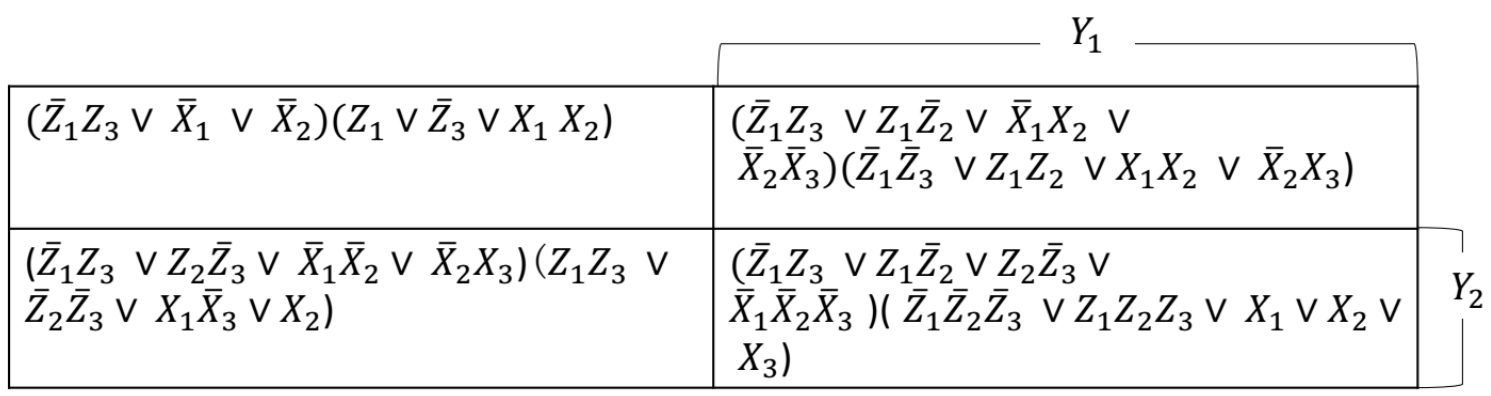

(b) $\quad h(\boldsymbol{X}, \boldsymbol{Y}, \boldsymbol{Z})$ with the NOR function replaced by its pos form

Fig. 2. A map expression of the function in Example 1 
International Journal of Mathematical, Engineering and Management Sciences

Vol. 3, No. 4, 404-428, 2018

https://dx.doi.org/10.33889/IJMEMS.2018.3.4-029

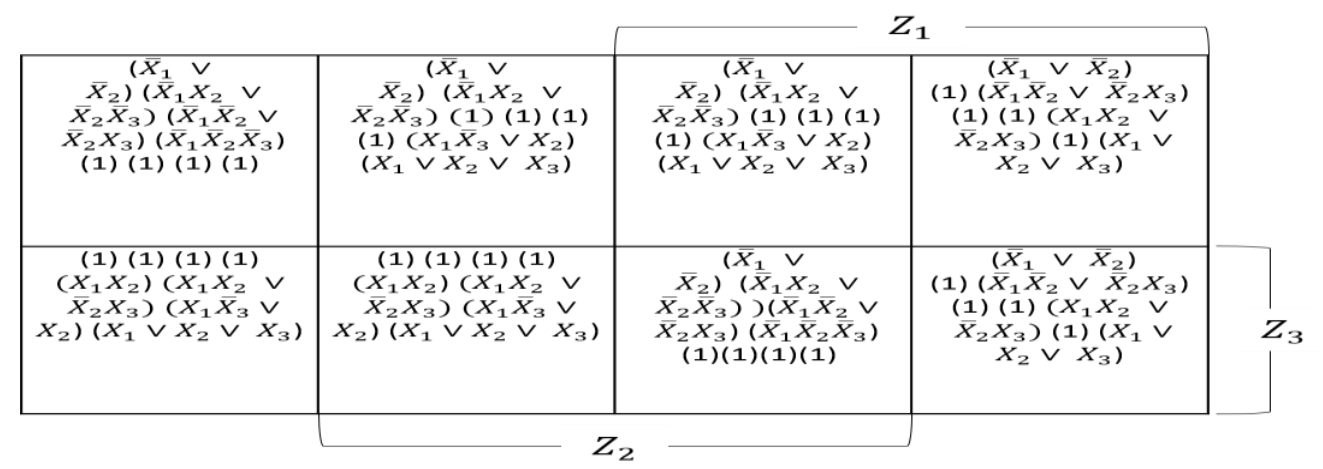

(a) $\quad g(\boldsymbol{X}, \boldsymbol{Z})$

\begin{tabular}{|l|c|c|c|}
\cline { 3 - 4 } $\bar{X}_{1} \bar{X}_{2} \bar{X}_{3}$ & $\bar{X}_{1} X_{2} \vee$ & $\bar{X}_{1} X_{2} \vee$ & $\bar{X}_{2} X_{3}$ \\
$X_{1} \bar{X}_{2} \bar{X}_{3}$ & $X_{1} \bar{X}_{2} \bar{X}_{3}$ & \\
\hline$X_{1} X_{2}$ & $X_{1} X_{2}$ & $\bar{X}_{1} \bar{X}_{2} \bar{X}_{3}$ & $\bar{X}_{2} X_{3}$ \\
\hline
\end{tabular}

(b) $\quad g(\boldsymbol{X}, \boldsymbol{Z})$

\begin{tabular}{|c|c|c|c|}
\hline $\bar{X}_{1} \bar{X}_{2} \bar{X}_{3}$ & $\begin{array}{c}\bar{X}_{1} X_{2} \bar{X}_{3} \vee \\
\bar{X}_{1} X_{2} X_{3} \vee \\
X_{1} \bar{X}_{2} \bar{X}_{3}\end{array}$ & $\begin{array}{c}\bar{X}_{1} X_{2} \bar{X}_{3} \vee \\
\bar{X}_{1} X_{2} X_{3} \vee \\
X_{1} \bar{X}_{2} \bar{X}_{3}\end{array}$ & $\begin{array}{c}X_{1} \bar{X}_{2} X_{3} \mathrm{v} \\
\bar{X}_{1} \bar{X}_{2} X_{3}\end{array}$ \\
\hline $\begin{array}{l}X_{1} X_{2} \bar{X}_{3} \\
\vee X_{1} X_{2} X_{3}\end{array}$ & $\begin{array}{l}X_{1} X_{2} \bar{X}_{3} \\
\vee X_{1} X_{2} X_{3}\end{array}$ & $\bar{X}_{1} \bar{X}_{2} \bar{X}_{3}$ & $\begin{array}{c}X_{1} \bar{X}_{2} X_{3} \mathrm{v} \\
\bar{X}_{1} \bar{X}_{2} X_{3}\end{array}$ \\
\hline
\end{tabular}

(c) $\quad g(\boldsymbol{X}, \boldsymbol{Z})$

Fig. 3. The function $g(\boldsymbol{X}, \boldsymbol{Z})$ in Example 1 
International Journal of Mathematical, Engineering and Management Sciences

Vol. 3, No. 4, 404-428, 2018

https://dx.doi.org/10.33889/IJMEMS.2018.3.4-029

\begin{tabular}{|c|c|c|c|}
\hline $\bar{X}_{1} \bar{X}_{2} \bar{X}_{3}\left(\bar{p}_{0}\right)$ & $\begin{array}{c}\bar{X}_{1} X_{2} \bar{X}_{3}\left(\bar{p}_{2}\right) \\
\vee \bar{X}_{1} X_{2} X_{3}\left(\bar{p}_{3}\right) \\
\vee X_{1} \bar{X}_{2} \bar{X}_{3}\left(\bar{p}_{4}\right)\end{array}$ & $\begin{array}{c}\bar{X}_{1} X_{2} \bar{X}_{3}\left(p_{2}\right) \\
\vee \bar{X}_{1} X_{2} X_{3}\left(p_{3}\right) \\
\vee X_{1} \bar{X}_{2} \bar{X}_{3}\left(p_{4}\right)\end{array}$ & $\begin{array}{c}\bar{X}_{1} \bar{X}_{2} X_{3}\left(\bar{p}_{1}\right) \vee \\
X_{1} \bar{X}_{2} X_{3}\left(\bar{p}_{5}\right)\end{array}$ \\
\hline $\begin{array}{c}X_{1} X_{2} \bar{X}_{3}\left(\bar{p}_{6}\right) \\
\vee X_{1} X_{2} X_{3}\left(\bar{p}_{7}\right)\end{array}$ & $\begin{array}{c}X_{1} X_{2} \bar{X}_{3}\left(p_{6}\right) \\
\vee X_{1} X_{2} X_{3}\left(p_{7}\right)\end{array}$ & $\bar{X}_{1} \bar{X}_{2} \bar{X}_{3}\left(p_{0}\right)$ & $\begin{array}{c}X_{1} \bar{X}_{2} X_{3}\left(p_{5}\right) \\
\vee \bar{X}_{1} \bar{X}_{2} X_{3}\left(p_{1}\right)\end{array}$ \\
\hline
\end{tabular}

$g(\boldsymbol{X} ; \boldsymbol{Z}, \boldsymbol{p})$

Fig. 4. The auxiliary function for Example 1

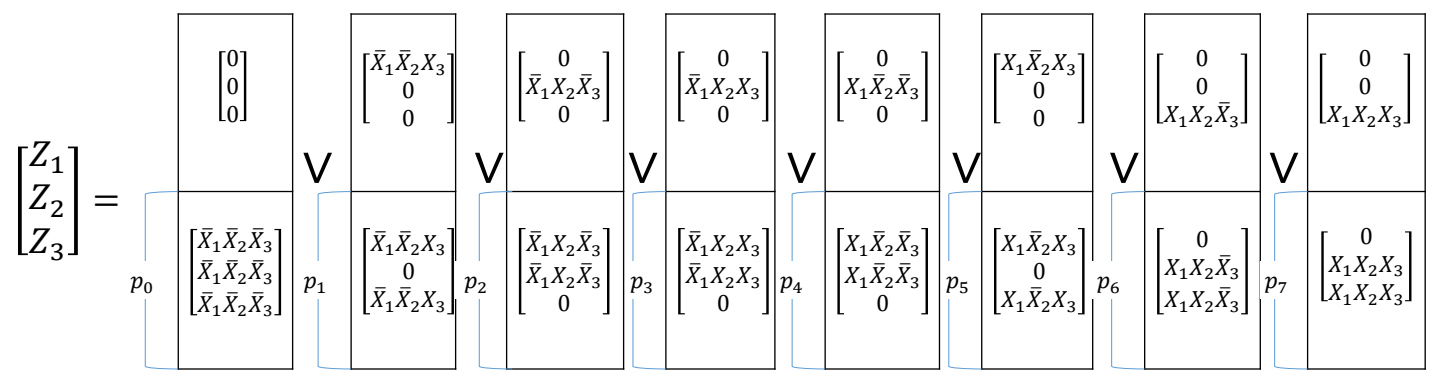

Fig. 5. A display of all 256 particular solutions of Example 1 as a disjunction of possible contributions of atoms $\mathrm{T}_{\mathrm{i}}(0 \leq \mathrm{i} \leq 7)$

\section{Example 2a}

Ledley $(1959,1960)$ described this example as one that illustrates a normally complicated solution. While this example has solutions such that $s=t$, a minor modification of it in Example $2 \mathrm{~b}$ produces a situation in which no solutions can be produced such that $s=t$. We start with two scalar functions $s$ and $t$, viz.

$s=\bar{Z}_{1} \bar{Z}_{2} \bar{Y}_{2} \vee Z_{1} \bar{Z}_{2} Y_{2} \vee \bar{Z}_{1} Z_{2}\left(Y_{1} \bar{Y}_{2} \vee \bar{Y}_{1} Y_{2}\right) \vee Z_{1} Z_{2} \bar{Y}_{1} \bar{Y}_{2}$

$t=\left(\bar{X}_{1} \bar{X}_{3} \vee \bar{X}_{2} X_{3}\right) \bar{Y}_{1} \bar{Y}_{2} \vee\left(\bar{X}_{1} \bar{X}_{3} \vee X_{1} X_{2}\right) Y_{1} \bar{Y}_{2} \vee\left(X_{1} \bar{X}_{3} \vee X_{2} X_{3}\right) \bar{Y}_{1} Y_{2} \vee\left(X_{1} \bar{X}_{2} \bar{X}_{3} \vee\right.$

$\left.\bar{X}_{1} X_{2} X_{3}\right) Y_{1} Y_{2}$

The function $h(\boldsymbol{X}, \boldsymbol{Y}, \boldsymbol{Z})$ equivalent to $(s \odot t)$ is expressed by the map in Fig. 6. Entries of the cells of this map are ANDed to form the function $g(\boldsymbol{X}, \boldsymbol{Z})$ in which $Y_{1}$ and $Y_{2}$ are suppressed. The natural map for $g(\boldsymbol{X}, \boldsymbol{Z})$ is gradually developed in Figs. 7(a)-7(d). The final map in Fig. 7(d) has entries in minterm form. It shows that each of the 8 atoms of $F B\left(X_{1}, X_{2}, X_{3}\right)$ appears once. Hence, 
International Journal of Mathematical, Engineering and Management Sciences

Vol. 3, No. 4, 404-428, 2018

https://dx.doi.org/10.33889/IJMEMS.2018.3.4-029

the number of particular solutions is $1^{8}=1$ and the consistency condition is the identity $(0=0)$. Therefore, the map can be used to represent the auxiliary function $G(\boldsymbol{X}, \boldsymbol{Z}, \boldsymbol{p})$ as well (since neither tagging each atom with 1 nor ORing each entry with $d(0)$ produces any change). The final answer for $Z(\boldsymbol{X})$ is unique and given by:

\begin{tabular}{|c|c|}
\hline$\left(\bar{Z}_{1} \bar{Z}_{2} \vee Z_{1} Z_{2}\right) \odot\left(\bar{X}_{1} \bar{X}_{3} \vee \bar{X}_{2} X_{3}\right)$ & $\left(\bar{Z}_{1} \bar{Z}_{2} \vee \bar{Z}_{1} Z_{2}\right) \odot\left(\bar{X}_{1} \bar{X}_{3} \vee X_{1} X_{2}\right)$ \\
\hline$\left(Z_{1} \bar{Z}_{2} \vee \bar{Z}_{1} Z_{2}\right) \odot\left(X_{1} \bar{X}_{3} \vee X_{2} X_{3}\right)$ & $Z_{1} \bar{Z}_{2} \odot\left(X_{1} \bar{X}_{2} \bar{X}_{3} \vee \bar{X}_{1} X_{2} X_{3}\right)$ \\
\hline
\end{tabular}

$h(\boldsymbol{X}, \boldsymbol{Y}, \boldsymbol{Z})$

Fig. 6. A VEKM representing a function equivalent to $(s \odot t)$ in Example 2a

\begin{tabular}{|c|c|}
\hline 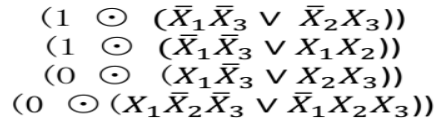 & 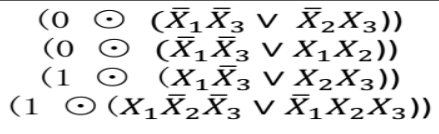 \\
\hline $\begin{array}{c}\left(0 \odot \odot\left(\bar{X}_{1} \bar{X}_{3} \vee \bar{X}_{2} X_{3}\right)\right) \\
\left(1-\odot\left(\bar{X}_{1} \bar{X}_{3} \vee X_{1} X_{2}\right)\right) \\
\left.\left(1-\odot{ }^{(} \bar{X}_{1} \bar{X}_{3} \vee X_{2} X_{3}\right)\right) \\
\left.\text { (0 } \odot\left(X_{1} \bar{X}_{2} \bar{X}_{3} \vee \bar{X}_{1} X_{2} X_{3}\right)\right)\end{array}$ & 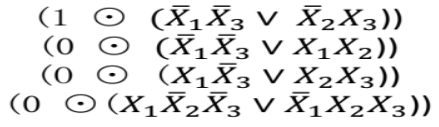 \\
\hline
\end{tabular}

(a) $\quad g(\boldsymbol{X}, \boldsymbol{Z})$

\begin{tabular}{|c|c|} 
& \\
& \\
$\left(\bar{X}_{1} \bar{X}_{3} \vee \bar{X}_{2} X_{3}\right)$ & $Z_{1}$ \\
$\left(\bar{X}_{1} \bar{X}_{3} \vee X_{1} X_{2}\right)$ & $\left(X_{1} \vee X_{3}\right)\left(X_{2} \vee \bar{X}_{3}\right)$ \\
$\left(\bar{X}_{1} \vee X_{3}\right)\left(\bar{X}_{2} \vee \bar{X}_{3}\right)$ & $\left(X_{1} \vee X_{3}\right)\left(\bar{X}_{1} \vee \bar{X}_{2}\right)$ \\
$\left(\bar{X}_{1} \vee X_{2} \vee X_{3}\right)\left(X_{1} \vee \bar{X}_{2} \vee \bar{X}_{3}\right)$ & $\left(X_{1} \bar{X}_{2} \bar{X}_{3} \vee \bar{X}_{1} X_{2} X_{3}\right)$ \\
\hline$\left(X_{1} \vee X_{3}\right)\left(X_{2} \vee \bar{X}_{3}\right)$ & $\left(\bar{X}_{1} \bar{X}_{3} \vee \bar{X}_{2} X_{3}\right)$ \\
$\left(\bar{X}_{1} \bar{X}_{3} \vee X_{1} X_{2}\right)$ & $\left(\bar{X}_{1} \vee X_{3}\right)\left(\bar{X}_{1} \vee \bar{X}_{2}\right)$ \\
$\left(X_{1} \bar{X}_{3} \vee X_{2} X_{3}\right)$ & $\left(\bar{X}_{1} \vee X_{3}\right)\left(\bar{X}_{2} \vee \bar{X}_{3}\right)$ \\
$\left(\bar{X}_{1} \vee X_{2} \vee X_{3}\right)\left(X_{1} \vee \bar{X}_{2} \vee \bar{X}_{3}\right)$ & $\left(\bar{X}_{1} \vee X_{2} \vee X_{3}\right)\left(X_{1} \vee \bar{X}_{2} \vee \bar{X}_{3}\right)$ \\
\hline
\end{tabular}

(b) $\quad g(\boldsymbol{X}, \boldsymbol{Z})$

\begin{tabular}{|c|c|}
\hline \multicolumn{1}{c|}{} & $Z_{1}$ \\
\hline $\bar{X}_{1} \bar{X}_{3}$ & $X_{1} \bar{X}_{2} \bar{X}_{3} \vee \bar{X}_{1} X_{2} X_{3}$ \\
\hline$X_{1} X_{2}$ & $\bar{X}_{2} X_{3}$ \\
\hline & \\
$Z_{2}$ \\
\hline
\end{tabular}


International Journal of Mathematical, Engineering and Management Sciences

Vol. 3, No. 4, 404-428, 2018

https://dx.doi.org/10.33889/IJMEMS.2018.3.4-029

(c) $\quad g(\boldsymbol{X}, \boldsymbol{Z})$

\begin{tabular}{|c|c|}
\hline $\begin{array}{c}\bar{X}_{1} X_{2} \bar{X}_{3} \\
\vee \bar{X}_{1} \bar{X}_{2} \bar{X}_{3}\end{array}$ & $\begin{array}{c}X_{1} \bar{X}_{2} \bar{X}_{3} \\
\vee \bar{X}_{1} X_{2} X_{3}\end{array}$ \\
\hline $\begin{array}{c}X_{1} X_{2} \bar{X}_{3} \\
\vee X_{1} X_{2} X_{3}\end{array}$ & $\begin{array}{c}\bar{X}_{1} \bar{X}_{2} X_{3} \\
\vee X_{1} \bar{X}_{2} X_{3}\end{array}$ \\
\hline
\end{tabular}

(d) $\quad g(\boldsymbol{X}, \boldsymbol{Z})$ or $G(\boldsymbol{X}, \boldsymbol{Z}, \boldsymbol{p})$

Fig. 7. Gradual development of the natural map for $g(\boldsymbol{X}, \boldsymbol{Z})$ obtained by suppressing $\boldsymbol{Y}$ from $h(\boldsymbol{X}, \boldsymbol{Y}, \boldsymbol{Z})$ in Fig. 6. The final map in $7(\mathrm{~d})$ also represents the auxiliary function $G(\boldsymbol{X}, \boldsymbol{Z}, \boldsymbol{p})$

$$
\begin{aligned}
Z_{1} & =X_{1} \bar{X}_{2} \bar{X}_{3} \vee \bar{X}_{1} X_{2} X_{3} \vee \bar{X}_{1} \bar{X}_{2} X_{3} \vee X_{1} \bar{X}_{2} X_{3} \\
& =X_{1} \bar{X}_{2} \vee \bar{X}_{1} X_{3} \\
Z_{2} & =X_{1} X_{2} \bar{X}_{3} \vee X_{1} X_{2} X_{3} \vee \bar{X}_{1} \bar{X}_{2} X_{3} \vee X_{1} \bar{X}_{2} X_{3} \\
& =X_{1} X_{2} \vee \bar{X}_{2} X_{3}
\end{aligned}
$$

\section{Example 2b}

Let us keep $t$ as given by (32) in Example 2a and augment $s$ therein by the term $\bar{Z}_{1} \bar{Z}_{2} \bar{Y}_{1}$ so as to become:

$s=\bar{Z}_{1} \bar{Z}_{2}\left(\bar{Y}_{1} \vee \bar{Y}_{2}\right) \vee Z_{1} \bar{Z}_{2} Y_{2} \vee \bar{Z}_{1} Z_{2}\left(Y_{1} \bar{Y}_{2} \vee \bar{Y}_{1} Y_{2}\right) \vee Z_{1} Z_{2} \bar{Y}_{1} \bar{Y}_{2}$

\begin{tabular}{|c|c|}
\cline { 2 - 2 } \multicolumn{1}{c|}{} & $Y_{1}$ \\
\hline$\left(\bar{Z}_{1} \bar{Z}_{2} \vee Z_{1} Z_{2}\right) \odot\left(\bar{X}_{1} \bar{X}_{3} \vee \bar{X}_{2} X_{3}\right)$ & $\left(\bar{Z}_{1} \bar{Z}_{2} \vee \bar{Z}_{1} Z_{2}\right) \odot\left(\bar{X}_{1} \bar{X}_{3} \vee X_{1} X_{2}\right)$ \\
\hline$\left(\bar{Z}_{1} \bar{Z}_{2} \vee \bar{Z}_{1} Z_{2} \vee Z_{1} \bar{Z}_{2}\right) \odot\left(X_{1} \bar{X}_{3} \vee X_{2} X_{3}\right)$ & $Z_{1} \bar{Z}_{2} \odot\left(X_{1} \bar{X}_{2} \bar{X}_{3} \vee \bar{X}_{1} X_{2} X_{3}\right)$ \\
& \\
\hline
\end{tabular}

$h(\boldsymbol{X}, \boldsymbol{Y}, \boldsymbol{Z})$

Fig. 8. A VEKM representing a function equivalent to $(s \odot t)$ in Example $2 \mathrm{~b}$ 
International Journal of Mathematical, Engineering and Management Sciences

Vol. 3, No. 4, 404-428, 2018

https://dx.doi.org/10.33889/IJMEMS.2018.3.4-029

\begin{tabular}{|c|c|}
\hline 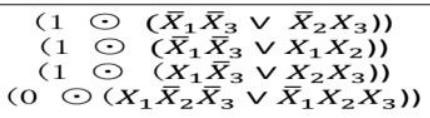 & 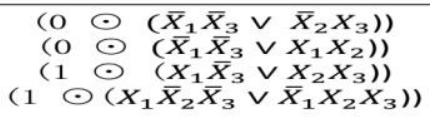 \\
\hline 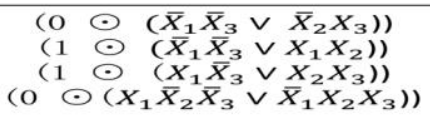 & 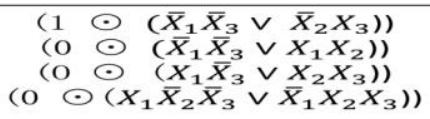 \\
\hline
\end{tabular}

(a) $\quad g(\boldsymbol{X}, \boldsymbol{Z})$

\begin{tabular}{|c|c|}
\hline $\begin{array}{c}\left(\bar{X}_{1} \bar{X}_{3} \vee \bar{X}_{2} X_{3}\right) \\
\left(\bar{X}_{1} \bar{X}_{3} \vee X_{1} X_{2}\right) \\
\left(X_{1} \bar{X}_{3} \vee X_{2} X_{3}\right) \\
\left(\bar{X}_{1} \vee X_{2} \vee X_{3}\right)\left(X_{1} \vee \bar{X}_{2} \vee \bar{X}_{3}\right)\end{array}$ & $\begin{array}{c}\left(X_{1} \vee X_{3}\right)\left(X_{2} \vee \bar{X}_{3}\right) \\
\left(X_{1} \vee X_{3}\right)\left(\bar{X}_{1} \vee \bar{X}_{2}\right) \\
\left(X_{1} \bar{X}_{3} \vee X_{2} X_{3}\right) \\
\left(X_{1} \bar{X}_{2} \bar{X}_{3} \vee \bar{X}_{1} X_{2} X_{3}\right)\end{array}$ \\
\hline $\begin{array}{c}\left(X_{1} \vee X_{3}\right)\left(X_{2} \vee \bar{X}_{3}\right) \\
\left(\bar{X}_{1} \bar{X}_{3} \vee X_{1} X_{2}\right) \\
\left(X_{1} \bar{X}_{3} \vee X_{2} X_{3}\right) \\
\left(\bar{X}_{1} \vee X_{2} \vee X_{3}\right)\left(X_{1} \vee \bar{X}_{2} \vee \bar{X}_{3}\right)\end{array}$ & $\begin{array}{c}\left(\bar{X}_{1} \bar{X}_{3} \vee\left(\bar{X}_{2} X_{3}\right)\right. \\
\left(X_{1} \vee X_{3}\right)\left(\bar{X}_{1} \vee \bar{X}_{2}\right) \\
\left(\bar{X}_{1} \vee X_{3}\right)\left(\bar{X}_{2} \vee \bar{X}_{3}\right) \\
\left(\bar{X}_{1} \vee X_{2} \vee X_{3}\right)\left(X_{1} \vee \bar{X}_{2} \vee \bar{X}_{3}\right)\end{array}$ \\
\hline
\end{tabular}

(b) $\quad g(\boldsymbol{X}, \boldsymbol{Z})$

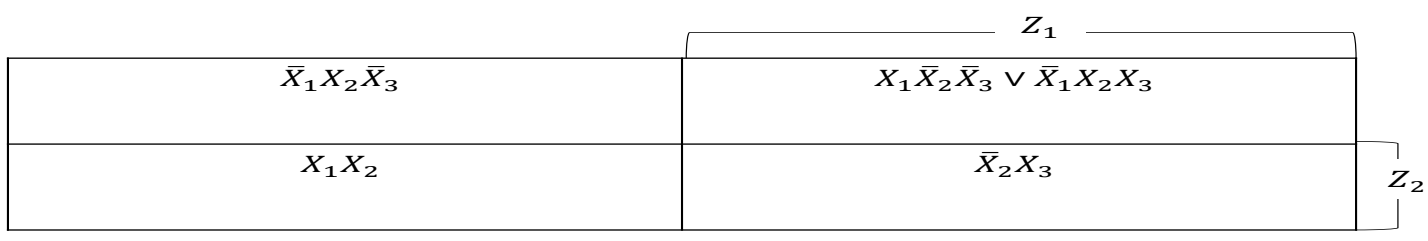

(c) $\quad g(\boldsymbol{X}, \boldsymbol{Z})$

\begin{tabular}{|c|c|}
\cline { 2 - 3 } \multicolumn{1}{c|}{} & $Z_{1}$ \\
\hline $\bar{X}_{1} X_{2} \bar{X}_{3}$ & $X_{1} \bar{X}_{2} \bar{X}_{3}$ \\
& $\vee \bar{X}_{1} X_{2} X_{3}$ \\
\hline$X_{1} X_{2} \bar{X}_{3}$ & \\
\hline$X_{1} X_{2} X_{3}$ & $\bar{X}_{1} \bar{X}_{2} X_{3}$ \\
$Z_{2}$ \\
\hline
\end{tabular}

(d) $\quad g(\boldsymbol{X}, \boldsymbol{Z})$

Fig. 9. Gradual development of the natural map for $g(\boldsymbol{X}, \boldsymbol{Z})$ obtained by suppressing $\boldsymbol{Y}$ from $h(\boldsymbol{X}, \boldsymbol{Y}, \boldsymbol{Z})$ in Fig. 8

We reproduce Figs. 6 and 7 modified as Figs. 8 and 9. In Fig. 9(d), only 7 atoms still appear (once each) while atom $\bar{X}_{1} \bar{X}_{2} \bar{X}_{3}$ is missing. Therefore, there is a single particular solution under the consistency condition $\bar{X}_{1} \bar{X}_{2} \bar{X}_{3}=0$, and the auxiliary function is given by Fig. 10. Hence the conditional solution is:

$$
\begin{aligned}
Z_{1} & =X_{1} \bar{X}_{2} \bar{X}_{3} \vee \bar{X}_{1} X_{2} X_{3} \vee \bar{X}_{1} \bar{X}_{2} X_{3} \vee X_{1} \bar{X}_{2} X_{3} \vee d\left(\bar{X}_{1} \bar{X}_{2} \bar{X}_{3}\right) \\
& =X_{1} \bar{X}_{2} \vee \bar{X}_{1} X_{3} \vee d\left(\bar{X}_{1} \bar{X}_{2} \bar{X}_{3}\right),
\end{aligned}
$$


International Journal of Mathematical, Engineering and Management Sciences

Vol. 3, No. 4, 404-428, 2018

https://dx.doi.org/10.33889/IJMEMS.2018.3.4-029

$$
\begin{aligned}
Z_{2} & =X_{1} X_{2} \bar{X}_{3} \vee X_{1} X_{2} X_{3} \vee \bar{X}_{1} \bar{X}_{2} X_{3} \vee X_{1} \bar{X}_{2} X_{3} \vee d\left(\bar{X}_{1} \bar{X}_{2} \bar{X}_{3}\right) \\
& =X_{1} X_{2} \vee \bar{X}_{2} X_{3} \vee d\left(\bar{X}_{1} \bar{X}_{2} \bar{X}_{3}\right) .
\end{aligned}
$$

\begin{tabular}{|c|c|}
\cline { 2 - 3 } \multicolumn{1}{c|}{} & $Z_{1}$ \\
\hline $\bar{X}_{1} X_{2} \bar{X}_{3}(1)$ & $X_{1} \bar{X}_{2} \bar{X}_{3}(1)$ \\
$\vee \mathrm{d}\left(\bar{X}_{1} \bar{X}_{2} \bar{X}_{3}\right)$ & $\vee \bar{X}_{1} X_{2} X_{3}(1)$ \\
& $\vee \mathrm{d}\left(\bar{X}_{1} \bar{X}_{2} \bar{X}_{3}\right)$ \\
\hline$X_{1} X_{2} \bar{X}_{3}(1)$ & $\bar{X}_{1} \bar{X}_{2} X_{3}(1)$ \\
$\vee X_{1} X_{2} X_{3}(1)$ & $\vee X_{1} \bar{X}_{2} X_{3}(1)$ \\
$\vee \mathrm{d}\left(\bar{X}_{1} \bar{X}_{2} \bar{X}_{3}\right)$ & $\vee \mathrm{d}\left(\bar{X}_{1} \bar{X}_{2} \bar{X}_{3}\right)$ \\
\hline
\end{tabular}

Fig. 10. The auxiliary function for Example 2b

\section{Example 3}

Suppose that both functions $s$ and $t$ are scalars of the form

$$
\begin{aligned}
& s(\boldsymbol{Z}, \boldsymbol{Y})=Z_{1} Y_{1} \vee \bar{Z}_{1} \bar{Y}_{1} \\
& t(\boldsymbol{X}, \boldsymbol{Y})=\bar{X}_{1} \bar{X}_{2} \vee \bar{X}_{1} Y_{1} \vee \bar{X}_{2} \bar{Y}_{1}
\end{aligned}
$$

Ledley (1960) labeled this example as one of no solutions. He said that subject to the condition $X_{1} \rightarrow \bar{X}_{2}$, solutions $Z_{1}=X_{2}$, and $Z_{2}=\bar{X}_{1}$ are antecedent solutions that make $s_{a} \rightarrow t$ but $s \neq t$. According to our method, we construct the function $h(\boldsymbol{X}, \boldsymbol{Y}, \boldsymbol{Z})$ according to (2b) in Fig. 11, and then suppress $Y_{1}$ to obtain $g(\boldsymbol{X}, \boldsymbol{Z})$, represented by the natural map in Fig.12(a). Out of the four atoms of $F B\left(X_{1}, X_{2}\right)$, the two atoms $X_{1} \bar{X}_{2}$ and $\bar{X}_{1} X_{2}$, make a single appearance in Fig. 12(b), while the two atoms $\bar{X}_{1} \bar{X}_{2}$ and $X_{1} X_{2}$ make no appearance therein. The number of particular solutions is $1 * 1=1$, and the consistency condition is $\left(\bar{X}_{1} \bar{X}_{2} \vee X_{1} X_{2}=0\right)$. We construct the auxiliary function $G(\boldsymbol{X}, \boldsymbol{Z}, \boldsymbol{p})$ via the map in Fig. 13 by tagging each of the asserted atoms $X_{1} \bar{X}_{2}$ and $\bar{X}_{1} X_{2}$ by 1 and adding each of the nullified atoms don't-care in each cell of the map of $G(\boldsymbol{X}, \boldsymbol{Z}, \boldsymbol{p})$. The parametric solution of $Z_{1}$ involves no parameters and constitutes a single particular solution given by

$$
Z_{1}=\bar{X}_{1} X_{2} \vee d\left(\bar{X}_{1} \bar{X}_{2} \vee X_{1} X_{2}\right)
$$

The expression above for $Z_{1}$ might be simplified to either $Z_{1}=\bar{X}_{1}$ or $Z_{1}=X_{2}$. These are not alternative solutions as claimed by Ledley (1960) but are the same solution under the above consistency condition which is equivalent to requiring that $\bar{X}_{1}=X_{2}$. Reading of the map in Fig. 13 (or complementing (40)) yields

$$
\bar{Z}_{1}=X_{1} \bar{X}_{2} \vee d\left(\bar{X}_{1} \bar{X}_{2} \vee X_{1} X_{2}\right)
$$

Substituting (40) and (41) in (38) produces

$$
\begin{aligned}
s & =\left(\bar{X}_{1} X_{2} Y_{1} \vee X_{1} \bar{X}_{2} \bar{Y}_{1}\right) \vee d\left(Y_{1} \vee \bar{Y}_{1}\right)\left(\bar{X}_{1} \bar{X}_{2} \vee X_{1} X_{2}\right) \\
& =\left(\bar{X}_{1} X_{2} Y_{1} \vee X_{1} \bar{X}_{2} \bar{Y}_{1}\right) \vee d\left(\bar{X}_{1} \bar{X}_{2} \vee X_{1} X_{2}\right)
\end{aligned}
$$


International Journal of Mathematical, Engineering and Management Sciences

Vol. 3, No. 4, 404-428, 2018

https://dx.doi.org/10.33889/IJMEMS.2018.3.4-029

which is exactly the same as $t$ in (39) under the auxiliary condition that $\left(\bar{X}_{1} \bar{X}_{2} \vee X_{1} X_{2}=0\right)$ or equivalently $X_{2}=\bar{X}_{1}$, or $X_{1}=\bar{X}_{2}$. This very simple example reveals many of the shortcoming in Ledley's method, including the inadequacy of splitting solutions into antecedence and consequence ones, and the difficulty of doing without the concept of a consistency condition.

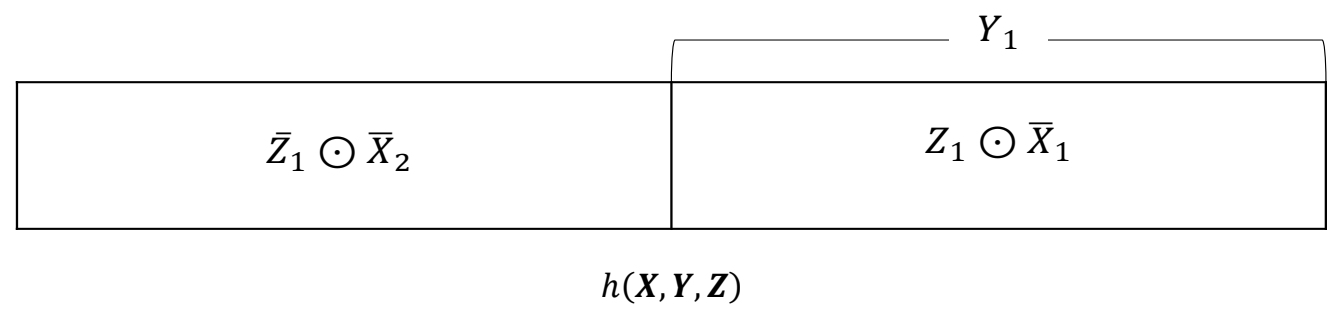

Fig. 11. Map representation of a function $h(\boldsymbol{X}, \boldsymbol{Y}, \boldsymbol{Z})$ whose assertion is equivalent to equality of $s$ and $t$ in Example 3

\begin{tabular}{|l|c|}
\multicolumn{1}{c|}{} & $Z_{1}$ \\
\hline$\left(1 \odot \bar{X}_{2}\right)$ & $\left(0 \odot \bar{X}_{2}\right)$ \\
$\left(0 \odot \bar{X}_{1}\right)$ & $\left(1 \odot \bar{X}_{1}\right)$ \\
\hline
\end{tabular}

(a) $\quad g(\boldsymbol{X}, \boldsymbol{Z})$

\begin{tabular}{|l|l|l|}
\multicolumn{1}{c|}{} & $Z_{1}$ \\
\hline$X_{1} \bar{X}_{2}$ & & $\bar{X}_{1} X_{2}$ \\
\hline & & \\
\hline & (b) $g(\boldsymbol{X}, \boldsymbol{Z})$
\end{tabular}

Fig. 12. Resultant of suppression of $Y_{1}$ in Example 3

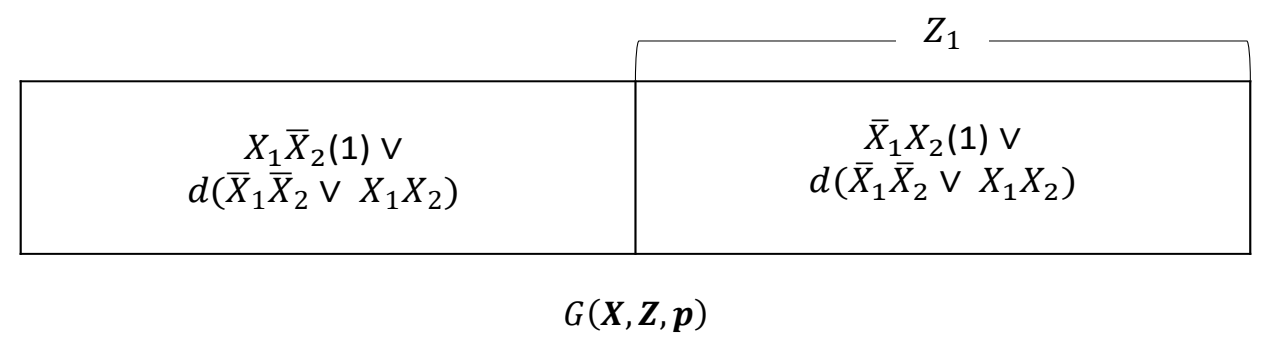

Fig. 13. The auxiliary function for Example 3 
International Journal of Mathematical, Engineering and Management Sciences

Vol. 3, No. 4, 404-428, 2018

https://dx.doi.org/10.33889/IJMEMS.2018.3.4-029

\section{Example 4}

Ledley (1960) required just a consequence solution $(t \rightarrow s)$ rather than a complete one $\{(s \rightarrow t) \wedge$ $(t \rightarrow s)\}$ for this example, where $s$ and $t$ are given by

$s=Z_{1}$

$t(\boldsymbol{X}, \boldsymbol{Y})=X_{1}\left(\bar{X}_{2} Y \vee X_{2} X_{3}\right) \vee \bar{X}_{1} \bar{Y}\left(X_{2} \bar{X}_{3} \vee \bar{X}_{2} X_{3}\right)$

An obvious (albeit trivial and non-genuine) solution for this problem is $Z_{1}=1$ (since 1 is implied by anything). We now use Figs. 14 and 15 to represent the pertinent maps, noting that now the $h(\boldsymbol{X}, \boldsymbol{Y}, \boldsymbol{Z})$ function represents $(t \rightarrow s)$ rather than $(s \odot t)$. The final map in Fig. 15(c) shows that all atoms are present, with 5 of them making a single appearance and 3 of them making a double appearance each. These three atoms are $T_{0}=\bar{X}_{1} \bar{X}_{2} \bar{X}_{3}$ whose instances are tagged by elements of the orthonormal set $\left\{p_{0}, \bar{p}_{0}\right\}, T_{3}=\bar{X}_{1} X_{2} X_{3}$ whose instances are tagged by elements of the orthonormal set $\left\{p_{3}, \bar{p}_{3}\right\}$, and $T_{6}=X_{1} X_{2} \bar{X}_{3}$ whose instances are tagged by elements of the orthonormal set $\left\{p_{6}, \bar{p}_{6}\right\}$, while the other 5 atoms are each tagged by (1) as shown in Fig. 16. The consistency condition is the identity $(0=0)$, and the number of particular solution is $1^{5} * 2^{3}=8$. The parametric solution is

$$
\begin{aligned}
& Z_{1}=\bar{X}_{1} \bar{X}_{2} \bar{X}_{3} p_{0} \vee \bar{X}_{1} X_{2} X_{3} p_{3} \vee X_{1} X_{2} \bar{X}_{3} p_{6} \vee \bar{X}_{1} \bar{X}_{2} X_{3} \vee \bar{X}_{1} X_{2} \bar{X}_{3} \vee X_{1} \bar{X}_{2} \bar{X}_{3} \vee X_{1} \bar{X}_{2} X_{3} \vee \\
& X_{1} X_{2} X_{3}
\end{aligned}
$$

where each of $p_{0}, p_{3}$, and $p_{6} \in\{0,1\}$ according to our novel method (Rushdi and Ahmad; 2017a, $2017 \mathrm{~b}$ ). If we employ the conventional method, we use a common value $p$ for each of $p_{0}, p_{3}$, and $p_{6}$, but this $p \in B_{256}=F B\left(X_{1}, X_{2}, X_{3}\right)$. Equation (45) can be conventionally written as a display of 8 particular solutions for $Z_{1}$ as follows.

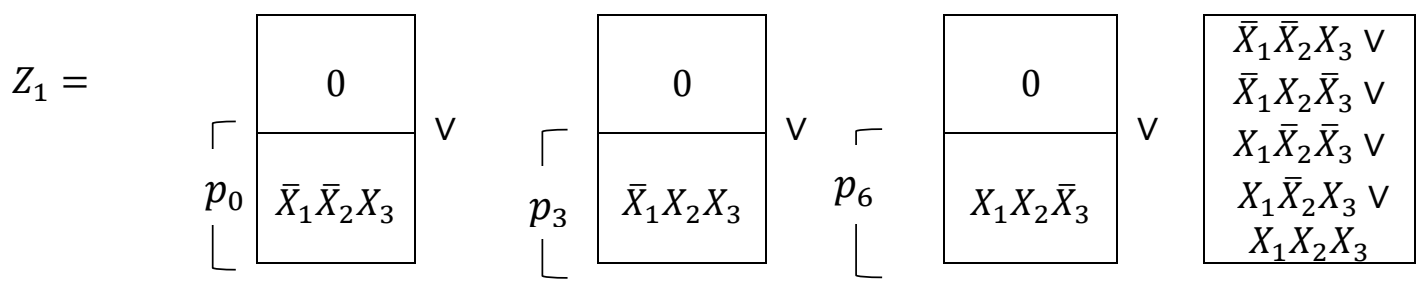

These 8 solutions are also displayed in Fig. 17. They include (beside the trivial solution $Z_{1}=1$ ), the three minimal genuine solutions identified by Ledley (1960).

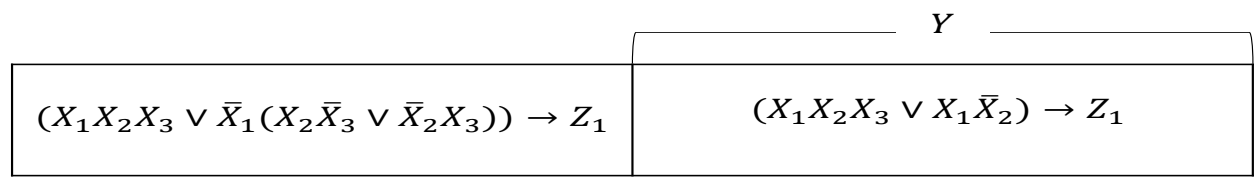

Fig. 14. The function $h(\boldsymbol{X}, \boldsymbol{Y}, \boldsymbol{Z})=t \rightarrow s$ in Example 4 
International Journal of Mathematical, Engineering and Management Sciences

Vol. 3, No. 4, 404-428, 2018

https://dx.doi.org/10.33889/IJMEMS.2018.3.4-029

$$
\begin{array}{|c|c|}
\cline { 2 - 2 } \multicolumn{1}{c|}{} & Z_{1} \\
\hline\left(\left(X_{1} X_{2} X_{3} \vee X_{1} \bar{X}_{2}\right) \rightarrow 0\right) & \left.\left.0 \bar{X}_{1}\right) \rightarrow 0\right) \\
\left(\left(X_{1} X_{2} X_{3} \vee \bar{X}_{1}\left(X_{2} \bar{X}_{3} \vee \bar{X}_{2} X_{3}\right)\right) \rightarrow 0\right) & \left(\left(X_{1} X_{2} X_{3} \vee X_{1} \bar{X}_{2}\right) \rightarrow 1\right) \\
& \left(\left(X_{1} X_{2} X_{3} \vee \bar{X}_{1}\left(X_{2} \bar{X}_{3} \vee \bar{X}_{2} X_{3}\right)\right) \rightarrow 1\right) \\
\hline
\end{array}
$$

(a) $\quad g(\boldsymbol{X}, \boldsymbol{Z})$

\begin{tabular}{|c|c|}
\cline { 2 - 2 } \multicolumn{1}{c|}{$\left(X_{2}\left(\bar{X}_{1} \vee \bar{X}_{3}\right) \vee \bar{X}_{1} \bar{X}_{2}\right)$} & $Z_{1}$ \\
\hline$\left(X_{1}\left(\bar{X}_{2} \vee \bar{X}_{3}\right) \vee \bar{X}_{1}\left(X_{2} X_{3} \vee \bar{X}_{2} \bar{X}_{3}\right)\right)$ & (1)(1) \\
\hline
\end{tabular}

(b) $\quad g(\boldsymbol{X}, \boldsymbol{Z})$

\begin{tabular}{|c|c|}
\hline $\begin{array}{c}\bar{X}_{1} \bar{X}_{2} \bar{X}_{3} \vee \\
\bar{X}_{1} X_{2} X_{3} \vee \\
X_{1} X_{2} \bar{X}_{3}\end{array}$ & $\begin{array}{c}\bar{X}_{1} \bar{X}_{2} \bar{X}_{3} \vee \\
\bar{X}_{1} X_{2} X_{3} \vee \\
X_{1} X_{2} \bar{X}_{3} \vee \\
\bar{X}_{1} \bar{X}_{2} X_{3} \vee \\
\bar{X}_{1} X_{2} \bar{X}_{3} \vee \\
X_{1} \bar{X}_{2} \bar{X}_{3} \vee \\
X_{1} \bar{X}_{2} X_{3} \vee \\
X_{1} X_{2} X_{3}\end{array}$ \\
\hline
\end{tabular}

(c) $\quad g(\boldsymbol{X}, \boldsymbol{Z})$

Fig. 15. Gradual development of the natural map for $g(\boldsymbol{X}, \boldsymbol{Z})$ obtained by suppressing $\boldsymbol{Y}$ from $h(\boldsymbol{X}, \boldsymbol{Y}, \boldsymbol{Z})$ in Fig. 14

\begin{tabular}{|c|c|}
\hline \multicolumn{1}{c|}{$Z_{1}$} \\
\cline { 2 - 2 } $\bar{X}_{1} \bar{X}_{2} \bar{X}_{3} \bar{p}_{0} \vee$ & $\bar{X}_{1} \bar{X}_{2} \bar{X}_{3} p_{0} \vee$ \\
$\bar{X}_{1} X_{2} X_{3} \bar{p}_{3} \vee$ & $\bar{X}_{1} X_{2} X_{3} p_{3} \mathrm{v}$ \\
$X_{1} X_{2} \bar{X}_{3} \bar{p}_{6}$ & $X_{1} X_{2} \bar{X}_{3} p_{6} \mathrm{v}$ \\
& $\bar{X}_{1} \bar{X}_{2} X_{3}(1) \mathrm{v}$ \\
& $\bar{X}_{1} X_{2} \bar{X}_{3}(1) \vee$ \\
& $X_{1} \bar{X}_{2} \bar{X}_{3}(1) \vee$ \\
& $X_{1} \bar{X}_{2} X_{3}(1) \vee$ \\
& $X_{1} X_{2} X_{3}(1)$ \\
\hline
\end{tabular}

Fig. 16. The auxiliary function for Example 4 
International Journal of Mathematical, Engineering and Management Sciences

Vol. 3, No. 4, 404-428, 2018

https://dx.doi.org/10.33889/IJMEMS.2018.3.4-029

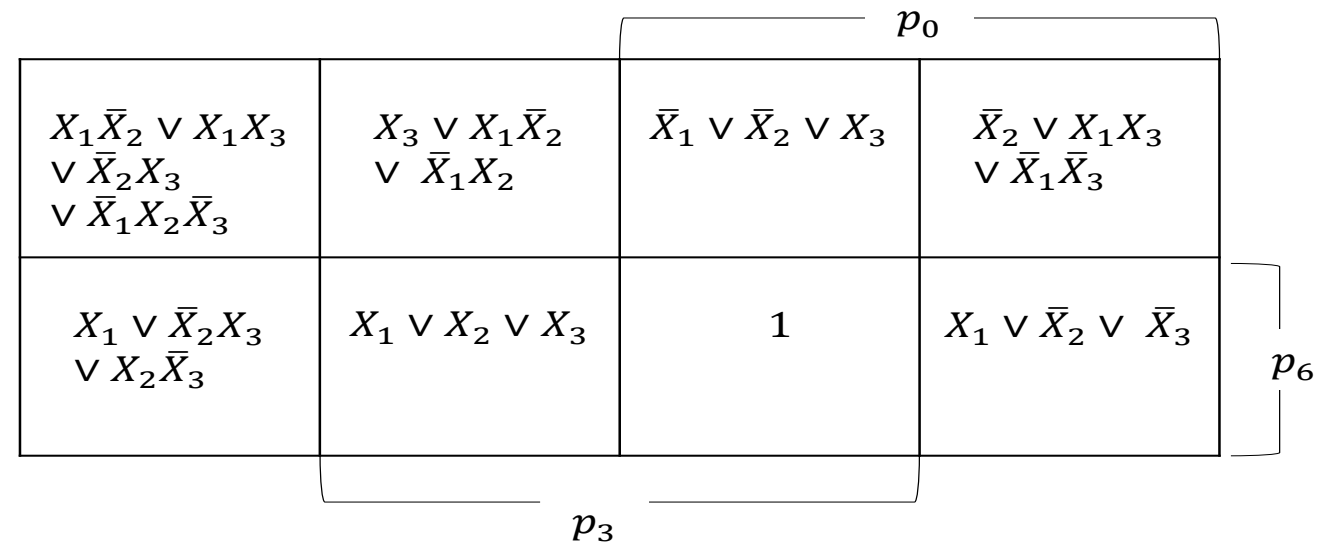

Fig. 17. Listing of all particular solutions of Example 4

\section{Example 5}

This example taken from Ledley (1959) illustrates the handling of genuinely vectorial function $\boldsymbol{S}$ and $\boldsymbol{t}$ (of dimensions $l=3>1$ ), given by

$$
\begin{aligned}
& s_{1}=\left(Z_{1} \vee \bar{Z}_{2}\right) \bar{Z}_{3} \vee Z_{1} \bar{Z}_{2} \\
& s_{2}=Z_{1} \bar{Z}_{3} \vee \bar{Z}_{1} Z_{3} \\
& s_{3}=Z_{2} \bar{Z}_{3} \vee \bar{Z}_{2} Z_{3} \\
& \text { and } \\
& t_{1}=X_{1}\left(\bar{X}_{2} \vee X_{3} \bar{X}_{4}\right) \vee \bar{X}_{2} X_{3} \\
& t_{2}=\left(\bar{X}_{1} \bar{X}_{3} \vee X_{1} X_{3}\right)\left(\bar{X}_{2} \vee \bar{X}_{4}\right) \\
& t_{3}=\bar{X}_{2}\left(\bar{X}_{3} \vee X_{1} X_{4}\right) \vee\left(X_{1} \vee X_{2}\right) X_{3} \bar{X}_{4}
\end{aligned}
$$

In (47c), we had to correct a typo in original expression given by Ledley (1959). This example lacks unwarranted $\boldsymbol{Y}$ variables, and hence we directly construct the function $g(\boldsymbol{X}, \boldsymbol{Z})$ such that

$g(\boldsymbol{X}, \boldsymbol{Z})=\bigwedge_{i=1}^{3}\left(s_{i}(\boldsymbol{Z}) \odot t_{i}(\boldsymbol{X})\right)$

This function is represented by the map in Fig. 18(b) inspired by the map in Fig. 18(a) and the facts that $(1 \odot t=t)$ and $(0 \odot t)=\bar{t}$. We compute the entries of the map in Fig. 18(b) via the conventional Karnaugh maps in Fig. 19. Our final result for $g(\boldsymbol{X}, \boldsymbol{Z})$ in Fig. 20 shows that there is a single appearance for each of the 16 atoms of $B_{512}=F B\left(X_{1}, X_{2}, X_{3}, X_{4}\right)$. Therefore, the consistency condition is the identity $(0=0)$, there is a single particular solution, and the map in Fig. 20 serves as well to represent the auxiliary function $G(\boldsymbol{X}, \boldsymbol{Z}, \boldsymbol{p})$. The final solution is 
International Journal of Mathematical, Engineering and Management Sciences

Vol. 3, No. 4, 404-428, 2018

https://dx.doi.org/10.33889/IJMEMS.2018.3.4-029

$\left[\begin{array}{l}Z_{1} \\ Z_{2} \\ Z_{3}\end{array}\right]=\left[\begin{array}{ll}X_{1} & \vee X_{2} X_{4} \\ X_{2} & \vee X_{1} X_{3} \\ \bar{X}_{3} & \vee X_{2} X_{4}\end{array}\right]$

in agreement with that found by Ledley (1959).

\begin{tabular}{|l|l|l|l|}
\cline { 3 - 4 } & \multicolumn{3}{c|}{$Z_{1}$} \\
\hline 011 & 001 & 111 & 110 \\
\hline \\
\hline
\end{tabular}

(a) $\quad s_{1} s_{2} s_{3}$

\begin{tabular}{|l|l|l|l|}
\cline { 3 - 4 } & \multicolumn{3}{c|}{$Z_{1}$} \\
\hline$t_{1} \bar{t}_{2} \bar{t}_{3}$ & $\bar{t}_{1} \bar{t}_{2} t_{3}$ & $t_{1} t_{2} t_{3}$ & $t_{1} t_{2} \bar{t}_{3}$ \\
\hline $\bar{t}_{1} t_{2} t_{3}$ & $\bar{t}_{1} t_{2} \bar{t}_{3}$ & $\bar{t}_{1} \bar{t}_{2} \bar{t}_{3}$ & $t_{1} \bar{t}_{2} t_{3}$ \\
$Z_{3}$ \\
\hline
\end{tabular}

(b) $\quad g(\boldsymbol{s}(\boldsymbol{Z}), \boldsymbol{t})$

Fig. 18. Map for the functions $s_{1}, s_{2}, s_{3}$, and $g(\boldsymbol{s}(\boldsymbol{Z}), \boldsymbol{t})$
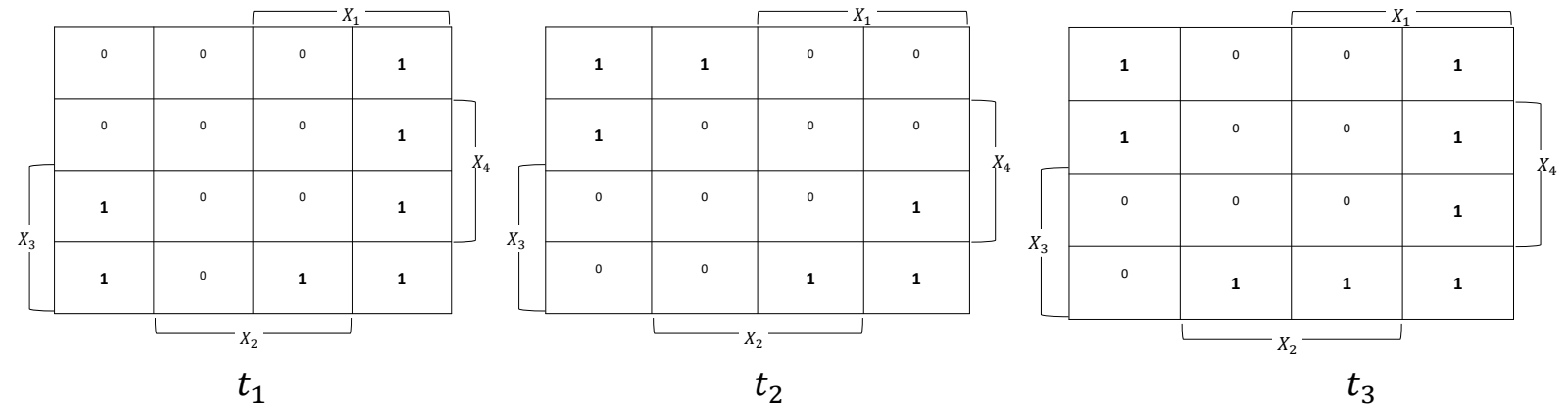
International Journal of Mathematical, Engineering and Management Sciences

Vol. 3, No. 4, 404-428, 2018

https://dx.doi.org/10.33889/IJMEMS.2018.3.4-029

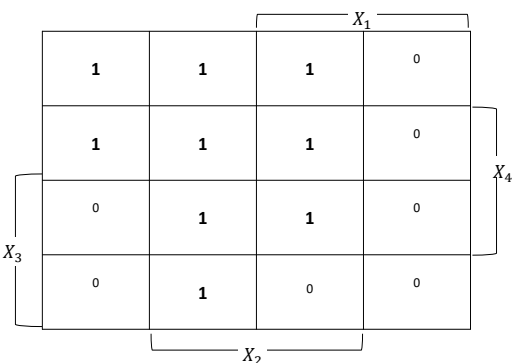

$\bar{t}_{1}$

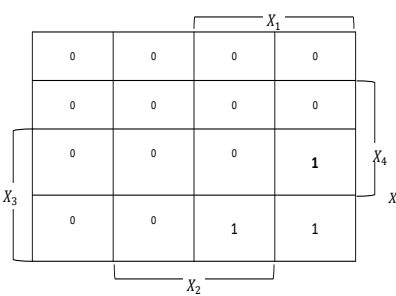

$t_{1} t_{2} t_{3}$

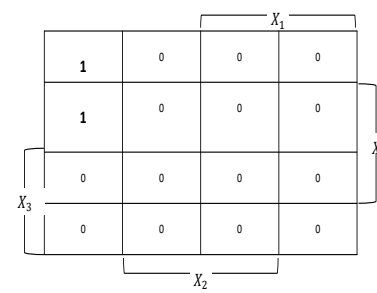

$\bar{t}_{1} t_{2} t_{3}$

$t_{1} t_{2} \bar{t}_{3}$

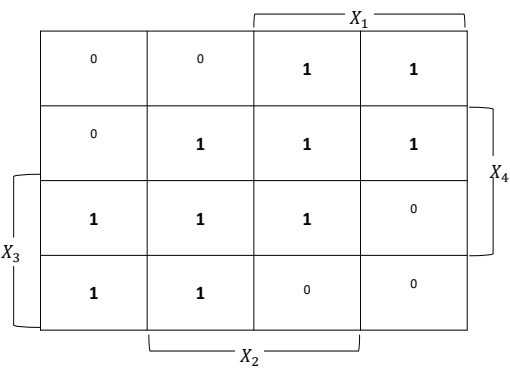

$\bar{t}_{2}$

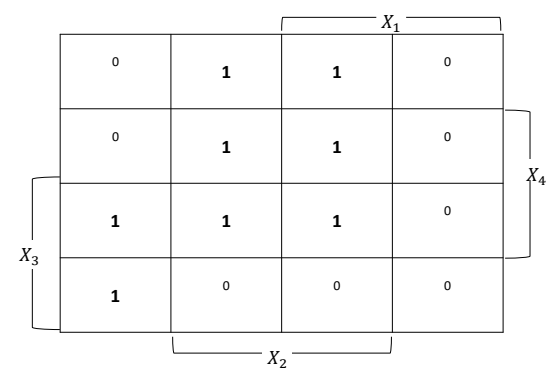

$\bar{t}_{3}$
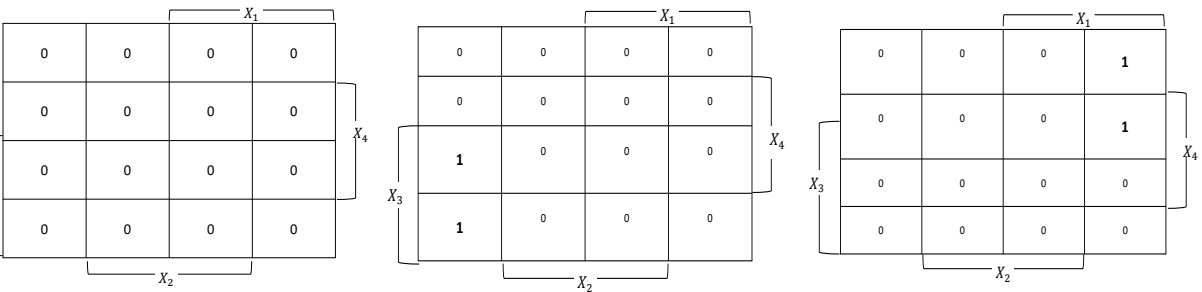

$t_{1} \bar{t}_{2} \bar{t}_{3}$

$t_{1} \bar{t}_{2} t_{3}$

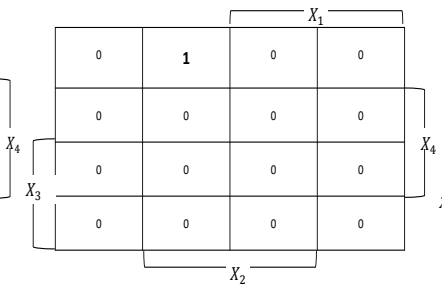

$\bar{t}_{1} t_{2} \bar{t}_{3}$

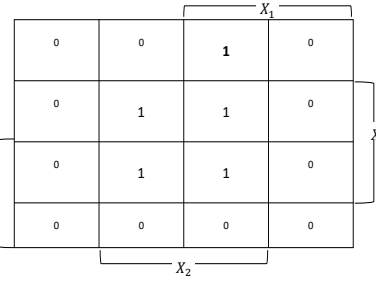

$\bar{t}_{1} \bar{t}_{2} \bar{t}_{3}$

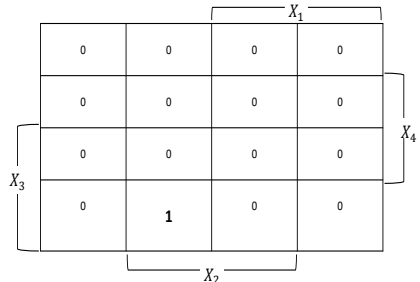

$\bar{t}_{1} \bar{t}_{2} t_{3}$

Fig. 19. Conventional Karnaugh maps used in the evaluation of entries in Fig. 18

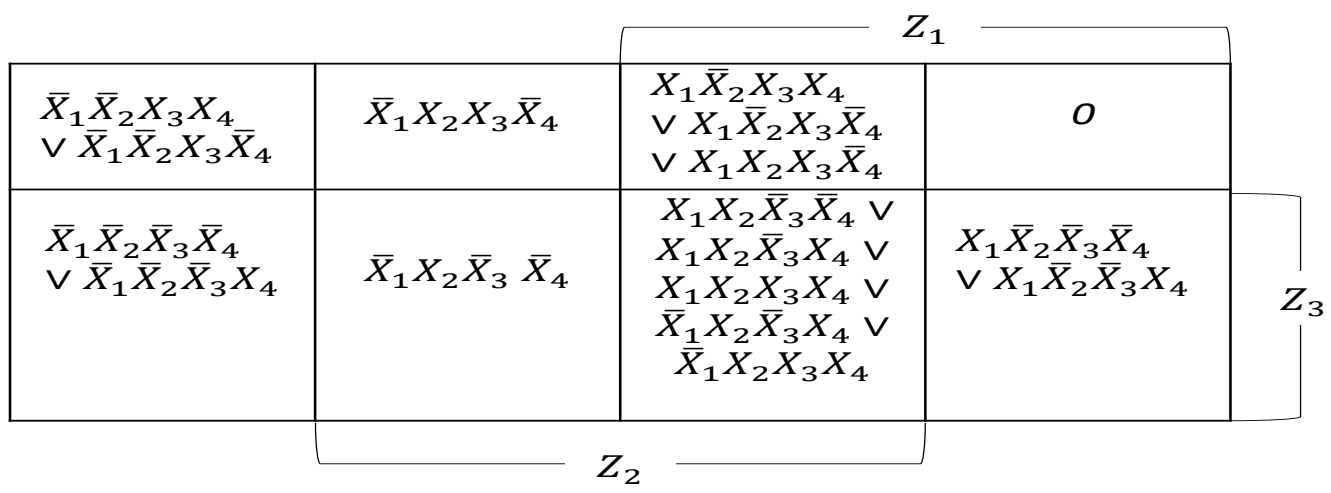

Fig. 20. Final map for both $g(\boldsymbol{X}, \boldsymbol{Z})$ and $G(\boldsymbol{X}, \boldsymbol{Z}, \boldsymbol{p})$ 
International Journal of Mathematical, Engineering and Management Sciences

Vol. 3, No. 4, 404-428, 2018

https://dx.doi.org/10.33889/IJMEMS.2018.3.4-029

\section{Conclusions}

This paper introduced dramatic improvements to the techniques used for handling an elementary problem of digital design. These improvements included (a) the use of a transparent algebraic representation all throughout the analysis instead of going to a cryptic numerical representation, (b) the solution of a single equation instead of solving two sets of equations at two sequential stages, (c) the use of powerful techniques of 'big' Boolean algebras to express outputs in terms of inputs, (d) a better conceptual understanding by imposing certain "consistency conditions" that allow complete conditional solutions to emerge instead of resorting to partial solutions or accepting that no solutions exist, and (e) the suppression of unwarranted or intermediary variables, right from the outset instead of making an extensive search as an afterthought. These improvements resulted in much faster (and occasionally corrected) solutions for the examples discussed herein.

\section{Acknowledgements}

The authors are greatly indebted to Engineer Mahmoud Ali Rushdi for the technical help he generously and proficiently offered during the preparation of this manuscript.

\section{References}

Ahmad, W., \& Rushdi, A. M. A. (2018). A new cryptographic scheme utilizing the difficulty of big Boolean satisfiability. International Journal of Mathematical, Engineering and Management Sciences, $3(1), 47-61$.

Baneres, D., Cortadella, J., \& Kishinevsky, M. (2009). A recursive paradigm to solve Boolean relations. IEEE Transactions on Computers, 58(4), 512-527.

Bell, N. R. (1968). A map method for the teaching of the fundamental concepts of compound-input logic circuits. IEEE Transactions on Education, E-11(3), 173-177.

Brown, F. M. (1970). Reduced solutions of Boolean equations. IEEE Transactions on Computers, C19(10), 976-981.

Brown, F. M. (1974). Equational logic. IEEE Transactions on Computers, C-23(12), 1228-1237.

Brown, F. M. (1975a). The constrained-input problem. IEEE Transactions on Computers, C-24(1), 102106.

Brown, F. M. (1975b). Equational realizations of switching functions. IEEE Transactions on Computers, C-24(11), 1054-1066.

Brown, F. M. (1990). Boolean reasoning: the logic of Boolean equations. Kluwer Academic Publishers, Boston, USA.

Brown, F. M. (2003). Boolean reasoning: the logic of Boolean equations. $2^{\text {nd }}$ Ed., Dover Publications, Mineola, NY, USA.

Brown, F. M. (2011). On the suppression of variables in Boolean equations. Discrete Applied Mathematics, $159(5), 255-258$.

Brown, S. D., \& Vranesic, Z. (2014). Fundamentals of digital logic with Verilog design. $3^{\text {rd }}$ Ed., McGrawHill, New York, NY, USA.

Cerny, E. (1976). Comments on "Equational logic". IEEE Transaction on Computers, C-25(1), 102-103.

Cerny, E., \& Marin, M. A. (1974). A computer algorithm for the synthesis of memoryless logic circuits. IEEE Transactions on Computers, C-23(5), 455-465. 
International Journal of Mathematical, Engineering and Management Sciences

Vol. 3, No. 4, 404-428, 2018

https://dx.doi.org/10.33889/IJMEMS.2018.3.4-029

Cerny, E., \& Marin, M. A. (1977). An approach to unified methodology of combinational switching circuits. IEEE Transaction on Computers, C-26(8), 745-756.

Jevons, W. S. (1872). On the inverse or inductive logical problem. Mem. Manchester Lit. Phil. Soc., 5, 119130.

Jevons, W. S. (1874). The Principles of Science: A treatise on logic and scientific method, England, London: MacMillan.

Knodel, O., Zabel, M., Lehmann, P., \& Spallek, R. G. (2014, November). Educating hardware designFrom Boolean equations to massively parallel computing systems. In Programmable Logic (SPL), 2014 IX Southern Conference on (pp. 1-6). IEEE.

Ledley, R. S. (1959). Boolean matrix equations in digital circuit design, IRE Transactions on Electronic Computers, EC-8 (6), 131-139.

Ledley, R. S. (1960). Digital computer and control engineering. McGraw-Hill, New York, NY, USA.

Poretsky, P. (1898). Sept lois fondamentales de la théorie des égalités logiques (Seven fundamental laws of the theory of logical inequalities), Bulletin de la Societe Physico-Mathematique de Kasan, 8, 33-103 and 129-216.

Rushdi, A. M. A. (1987). Improved variable-entered Karnaugh map procedures. Computer and Electrical Engineering, 13(1), 41-52.

Rushdi, A. M. A. (2001). Using variable-entered Karnaugh maps to solve Boolean equations. International Journal of Computer Mathematics, 78(1), 23-38.

Rushdi, A. M. A. (2004). Efficient solution of Boolean equations using variable-entered Karnaugh maps. Journal of King Abdulaziz University: Engineering Sciences, 15(1), 105-121.

Rushdi, A. M. A. (2012). A comparison of algebraic and map methods for solving general Boolean equations. Journal of Qassim University: Engineering and Computer Sciences, 5(2), 147-173.

Rushdi, A. M. A. (2018a). Utilization of Karnaugh maps in multi-value qualitative comparative analysis. International Journal of Mathematical, Engineering and Management Sciences, 3(1), 28-46.

Rushdi, A. M. A. (2018b). Handling generalized type-2 problems of digital circuit design via the variableentered Karnaugh map. International Journal of Mathematical, Engineering and Management Sciences (accepted).

Rushdi, A. M. A., \& Ahmad, W. (2016). Finding all solutions of the Boolean satisfiability problem, if any, via Boolean-equation solving. Journal of King Abdulaziz University: Engineering Sciences, 27(1), 1934.

Rushdi, A. M. A., \& Ahmad, W. (2017a). A novel method for compact listing of all particular solutions of a system of Boolean equations. British Journal of Mathematics \& Computer Science, 22(6), 1-18.

Rushdi, A. M. A., \& Ahmad, W. (2017b). Satisfiability in big Boolean algebras via Boolean-equation solving. Journal of King Abdulaziz University: Engineering Sciences, 28(1), (in press).

Rushdi, A. M. A., \& Ahmad, W. (2018). A comparison of two novel methods for handling a certain class of problems of digital circuit design. Journal of Qassim University: Engineering and Computer Sciences, $11(1)$, (in press).

Rushdi, A. M. A., \& Albarakati, H. M. (2014). Prominent classes of the most general subsumptive solutions of Boolean equations. Information Sciences, 281(x), 53-65.

Rushdi, A. M. A., \& Ba-Rukab, O. M. (2003). Low-cost design of multiple-output switching circuits using map solutions of Boolean equations. Umm Al-Qura University Journal of Science-MedicineEngineering, 15(2), 59-79. 
International Journal of Mathematical, Engineering and Management Sciences

Vol. 3, No. 4, 404-428, 2018

https://dx.doi.org/10.33889/IJMEMS.2018.3.4-029

Rushdi, A. M. A., \& Ba-Rukab, O. M. (2017). Map calculation of the Shapley-Shubik voting powers: an example of the European Economic Community. International Journal of Mathematical, Engineering and Management Sciences, 2(1) 17-29.

Rushdi, A. M., \& Al-Yahya, H. A. (2000). A Boolean minimization procedure using the variable-entered Karnaugh map and the generalized consensus concept. International Journal of Electronics, 87(7) 769794.

Rushdi, A. M., \& Al-Yahya, H. A. (2001). Further improved variable-entered Karnaugh map procedures for obtaining the irredundant forms of an incompletely-specified switching function. Journal of King Abdulaziz University: Engineering Sciences, 13(1), 111-152.

Rushdi, A. M., \& Amashah, M. H. (2010). Parametric general solutions of Boolean equations via variableentered Karnaugh maps. Journal of Qassim University: Engineering and Computer Sciences, 3(1), 5971.

Rushdi, A. M., \& Amashah, M. H. (2011). Using variable-entered Karnaugh maps to produce compact parametric general solutions of Boolean equations. International Journal of Computer Mathematics, 88(15), 3136-3149.

Rushdi, A. M., \& Amashah, M. H. (2012). Purely-algebraic versus VEKM methods for solving big Boolean equations. Journal of King Abdulaziz University: Engineering Sciences, 23(2), 75-85.

Steinbach, B., \& Posthoff, C. (2003, February). Optimal design by resolving Boolean equations. In $C A D$ Systems in Microelectronics, 2003. CADSM 2003. Proceedings of the 7th International Conference. The Experience of Designing and Application of (pp. 454-457). IEEE.

Venn, J. (1894). Symbolic logic. England, London: MacMillan. 